\title{
Suppressing or blocking Fis1 reverses diabetic endothelial dysfunction in human resistance arteries
}

\author{
Authors: Mamatha Kakarla ${ }^{1} \uparrow$, John M. Egner ${ }^{2} \uparrow$, Jingli Wang, ${ }^{1}$ Megan C. Harwig ${ }^{2}$, \\ Kelsey A. Meacham ${ }^{2}$, Venkata K. Puppala ${ }^{1}$, Benjamin C. Hofeld ${ }^{1}$, Leggy A. Arnold ${ }^{3}$, \\ David Z. Trykall², R. Blake Hill ${ }^{2 *}$, and Michael E. Widlansky ${ }^{1,4^{*}}$
}

\author{
Affiliations: \\ ${ }^{1}$ Department of Medicine, Division of Cardiovascular Medicine, Medical College of \\ Wisconsin, Milwaukee, WI. \\ ${ }^{2}$ Department of Biochemistry, Medical College of Wisconsin, Milwaukee, WI. \\ ${ }^{3}$ Department of Chemistry \& Biochemistry and Milwaukee Institute for Drug Discovery, \\ University of Wisconsin-Milwaukee, Milwaukee, WI. \\ ${ }^{4}$ Department of Pharmacology, Division of Cardiovascular Medicine, Medical College of \\ Wisconsin, Milwaukee, WI. \\ $\dagger$ These authors contributed equally to this work. \\ *Corresponding authors. Email: mwidlans@mcw.edu (M.E.W.); rbhill@mcw.edu \\ (R.B.H.)
}

One Sentence Summary: Micro- and macro-vascular complications in type 2 diabetes mellitus (T2DM) continue to be major health burdens in the United States and we identify a new therapeutic route to treatment by showing that either a novel peptide inhibitor, or genetic silencing, of mitochondrial fission protein 1 reverses poor vasodilation of human resistance arteries from diabetic patients.

\begin{abstract}
Mitochondrial dysfunction drives the development of vascular endothelial dysfunction in type 2 diabetes (T2DM) with increased fragmentation of mitochondrial networks from increased Fis 1 expression; whether suppressing or blocking Fis1 expression or activity can reverse endothelial dysfunction remains unknown. To address this question, resistance arterioles from healthy humans and those with T2DM were transfected with Fis1 siRNA and exposed to normal glucose, low glucose or high glucose conditions. Fis1 knockdown improved endothelium dependent vasodilation in T2DM arterioles, and blocked high- and low-glucose impairment in healthy vessels. Fis1 knockdown preserved NO bioavailability and improved endothelial layer integrity of cells exposed to high or low glucose. Fis1 knockdown had no significant effect on the expression of other mitochondrial dynamics or autophagy proteins, and had no effect on endothelial cell metabolism suggesting its suitability for pharmacological inhibition. For this, we designed pep213 to inhibit Fis1 activity $(K d \sim 3-7 \mu \mathrm{M})$ and demonstrate its specificity by NMR. Application of a cell permeant pep213 improved endotheliumdependent vasodilation in T2DM and non-T2DM vessels exposed to high glucose in an
\end{abstract}


NO-dependent manner suggesting that targeting Fis 1 may reduce vascular complications in T2DM.

\section{Introduction}

Impaired vascular endothelial function predates the development of macrovascular and microvascular disease in patients with type 2 diabetes (T2DM). Emerging data implicate abnormal mitochondrial form and function in the development of vascular endothelial dysfunction in humans with $\operatorname{T2DM}(1,2)$. Mitochondria in the endothelium of individuals with T2DM produce excessive superoxide. The excess superoxide is driven, in part, by greater polarization of the mitochondrial inner membrane (2). Excessive mitochondrial reactive oxygen species (mtROS) production in the endothelium activates critical epigenetic changes and cell signaling pathways that lead to endothelial inflammation and vascular dysfunction (3). We have previously shown that mitochondrial-targeted antioxidants or pharmacological agents dosed to partially depolarize the mitochondrial inner membrane reverse impaired endothelium-dependent vasodilation in resistance arterioles from humans with $\mathrm{T} 2 \mathrm{DM}(2,4)$.

Unfortunately, the phase 3 clinical trials of anti-oxidant therapeutic approaches to prevent and treat vascular diseases have failed to verify positive effects seen in smaller physiological and/or non-randomized studies $(5,6)$, and current pharmacological agents that target the mitochondrial inner membrane have toxicity profiles that preclude their clinical use (7). Targeting proteins involved in mitochondrial fission represent a promising alternative approach based on prior work demonstrating (1) increased expression of mitochondrial fission 1 protein (Fis1) in endothelial cells obtained from humans with type 2 diabetes; (2) molecular knockdown of Fis 1 or dynamin-related protein 1 (Drp1, which may bind Fis1 to induce mitochondrial fission) expression blocks high-glucose induced increases in mitochondrial superoxide production and impairment of phosphorylation of endothelium-derived nitric oxide synthase (eNOS) at its Ser1177 activation site; and (3) pharmacological and molecular knockdown of Drp1 reverse lowglucose induced endothelial dysfunction in human resistance arterioles $(1,8)$.

Fis1 as a pharmacological target is of particular interest given its role in mitochondrial dynamics appears to be most highly active in the setting of pathological stimuli like hypoxemia and hyperglycemia (9-12). In this set of investigations, we tested whether knockdown of Fis 1 expression reversed impaired endothelium-dependent vasodilation and nitric oxide (NO) production in resistance vessels from patients with type 2 diabetes and in vessels from healthy individuals exposed acutely to high and low glucose concentrations. Additionally, we determined the effect of Fis 1 knockdown on endothelial cell barrier function, oxygen consumption, and glycolysis under high or low glucose conditions. Finally, we designed and tested whether a novel peptide engineered to bind to Fis 1 and block Fis-1 mediated fission favorably impacted endothelium-dependent vasodilation of small resistance arteries from humans with T2DM and from healthy human vessels exposed to high glucose concentrations. Our findings support that pharmacological targeting of Fis1 might provide a useful therapeutic avenue for the challenge of vascular disease in T2DM.

\section{Results}




\section{Study Subject Characteristics}

A total of 67 subjects (14 T2DM, 53 healthy controls) were recruited. A full description of the characteristics of subjects who participated in this project's studies is presented in Table 1. Our healthy subjects were significantly younger than the subjects with T2DM ( $39 \pm 15$ vs. $55 \pm 12$ years, $\mathrm{P}=0.0002)$. Our healthy control subject group had a significantly lower body mass index, waist circumference, systolic blood pressure, fasting glucose, and glycosylated hemoglobin. Healthy subjects had higher HDL cholesterol levels as well. None of the healthy subjects were taking chronic cardiometabolic medications. We additionally present the subject characteristics for each investigation involving human vessels (Supplemental Tables 1-5).

\section{Suppressing Fis 1 improves endothelium-dependent vasodilation and NO bioavailability in human vessels}

We first measured vasodilation in healthy human resistance vessels by videomicroscopy incubated with glucose at concentrations experienced by T2DM patients (Figure 1A-B). Consistent with our earlier findings, both high (33mM) and low $(2.5 \mathrm{mM})$ glucose blunted the endothelium-dependent vasodilatory response to acetylcholine that appeared primarily related to eNOS activity based on inhibition with LNAME. To address whether the mitochondrial fission protein Fis 1 might be involved in vasodilatory impairment, we transfected the healthy vessels with Fis1 siRNA that leads to approximately 30\% reduction in Fis1 mRNA (Supplemental Figure 1). In these healthy human resistance vessels, transfection of Fis1 siRNA, but not a scrambled control, protected against high and low glucose-induced impairments in endothelium-dependent vasodilation (Figure 1A-B). Administration of L-NAME completely blocked this protective effect in both cases suggesting that Fis1 siRNA associated improvements are eNOS-dependent.
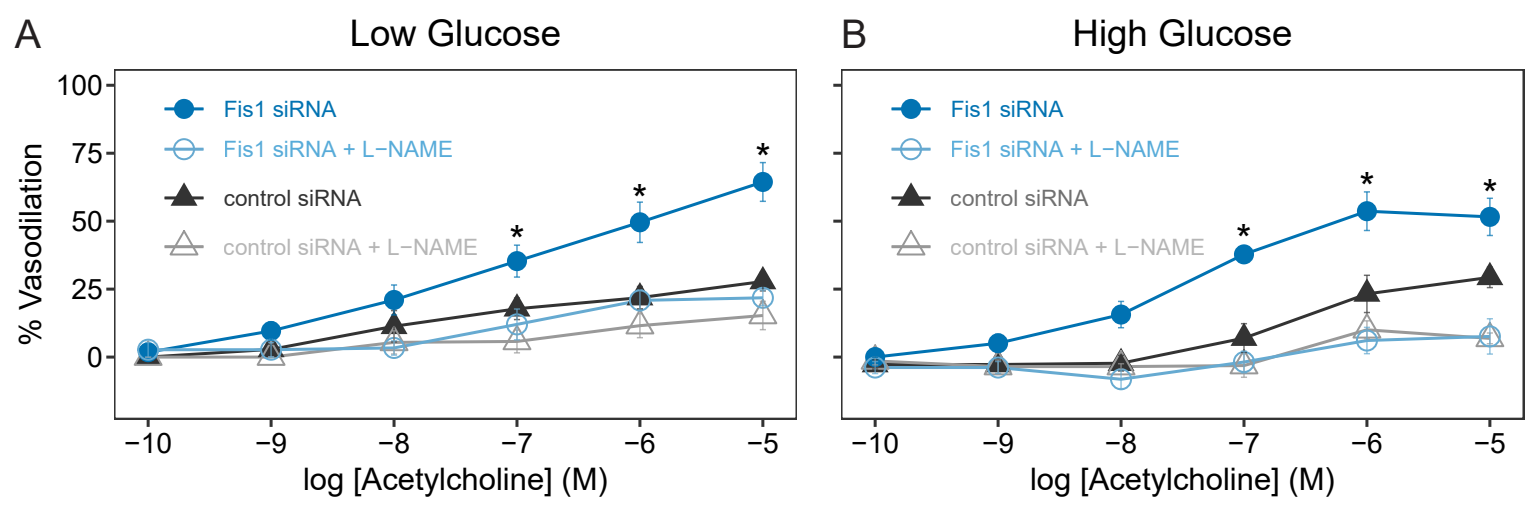

Figure 1: Suppression of Fis1 expression in healthy human vessels under low $(2.5 \mathrm{mM})$ and high $(33 \mathrm{mM})$ glucose conditions improves endothelium-dependent vasodilation. Human resistance vessels isolated from normal healthy subjects were treated with Fis 1 siRNA and vasodilation measured by videomicroscopy upon acetylcholine treatment. The impaired vasodilation caused by incubation with either low (A, $2.5 \mathrm{mM}, 2 \mathrm{hrs}$ ) or high (B, $33 \mathrm{mM}, 6 \mathrm{hrs}$ ) glucose conditions was improved upon Fis 1 siRNA treatment. For low glucose, $\mathbf{A}, \mathrm{P}=0.0008$ overall, $* \mathrm{P} \leq 0.0003$ at indicated acetylcholine doses for control siRNA vs Fis1 siRNA, N=6 subjects. For high glucose, B, $\mathrm{P}=0.0002$ overall, ${ }^{*} \mathrm{P} \leq 0.0002$ at indicated acetylcholine doses for control siRNA vs Fis 1 
siRNA, N=6 subjects. Treatment with $100 \mu \mathrm{M}$ L-NAME for 30 minutes and remeasurement abolishes vasodilatory improvement under Fis1 knockdown conditions under both glucose conditions (for low glucose, $\mathrm{P}=0.0002$ for Fis 1 siRNA vs Fis 1 siRNA+L-NAME; for high glucose, $\mathrm{P}<0.0001$ for Fis 1 siRNA vs siFis $1+\mathrm{L}-\mathrm{NAME}$ and $\mathrm{P}=0.002$ for control siRNA vs control siRNA+L-NAME). Uncertainties are standard error of the mean.

Consistent with this interpretation, Fis1 siRNA transfection also significantly increased the fluorescence of NO-sensitive dye DAF-2 DA in vessels from healthy individuals exposed to high glucose [Figure 2A; $n=9, \mathrm{P}=0.04$ for Fis 1 siRNA versus all other exposures (scrambled control, scrambled control + LNAME, and Fis1 siRNA + LNAME) ] and low glucose (Figure 2B, $\mathrm{n}=8, \mathrm{P}=0.01$ for Fis 1 siRNA vs. all other exposures). L-NAME completely abolished the increased in DAF-2 DA fluorescence in both cases. These data support the concept that Fis1 silencing can improve vasodilation in an NO-dependent manner under stress conditions experienced by T2DM patients. We next asked whether Fis 1 siRNA treatment could improve vasodilatory activity in resistance vessels from T2DM patients. Treatment with Fis 1 siRNA reversed impaired endothelium-dependent vasodilation (Figure 3; $n=6, P=0.002$ overall). As with vessels from healthy subjects, the favorable effect of Fis1 siRNA transfection on vessels from diabetic subjects was completely attenuated by L-NAME. Fis1 siRNA transfection did not impact the papaverine effect on vasodilation in any of these studies indicating that the effects were endothelial cell specific (data not shown). We had similar findings with genetic silencing of Drp1 in vessels from healthy and diabetic individuals (Supplemental Figures 2-4) consistent with excessive mitochondrial fission playing an important role in impairing NO-dependent vasodilation in the diabetic endothelium.
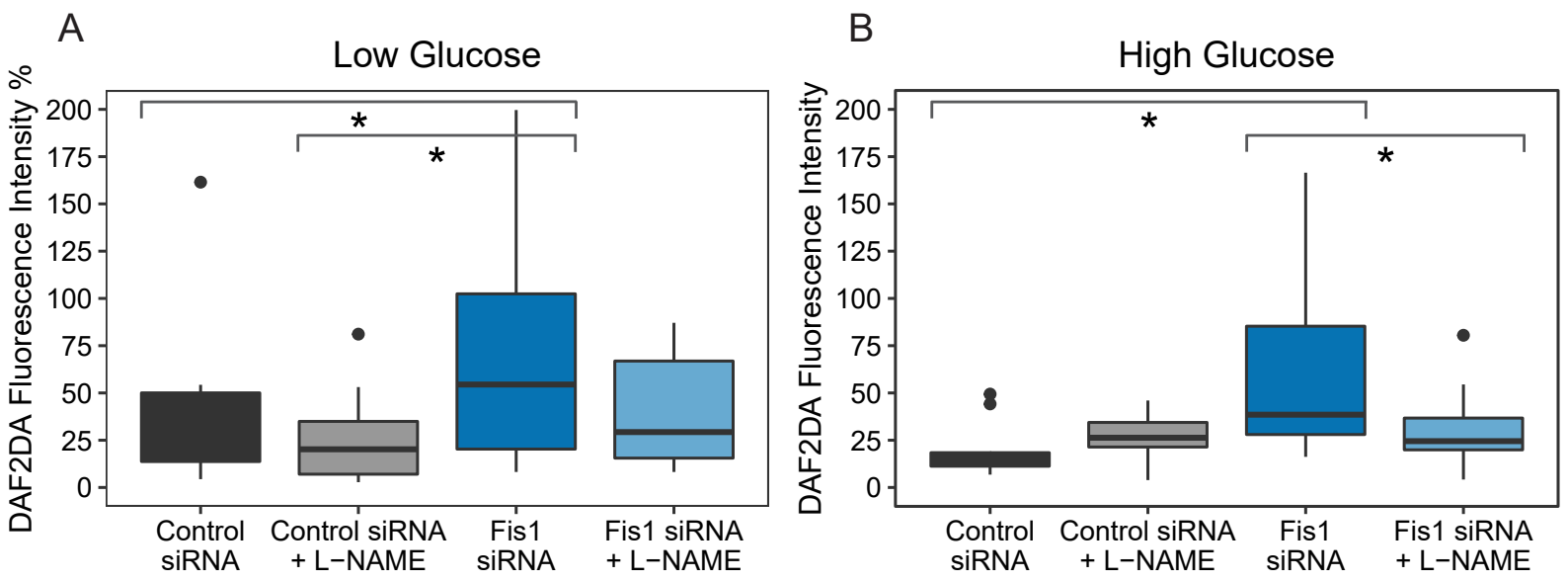

Figure 2: Suppression of Fis1 expression in healthy human vessels under low (2.5 $\mathrm{mM})$ and high $(33 \mathrm{mM})$ glucose conditions increases NO bioavailability. Human resistance vessels isolated from normal healthy subjects were treated with Fis 1 siRNA and the fluorescent NO marker DAF-2 DA measured under low (A, $2.5 \mathrm{mM}, 2 \mathrm{hrs})$ or high (B, $33 \mathrm{mM}, 6 \mathrm{hrs})$ glucose conditions upon Fis1 siRNA treatment. For low glucose conditions, $\mathbf{A}, \mathrm{n}=8$, overall $\mathrm{P}=0.01 ; \mathrm{P}=0.03$ for control siRNA vs. Fis 1 siRNA; $\mathrm{P}=0.003$ 
for control siRNA+L-NAME vs Fis 1 siRNA; $\mathrm{P}=0.03$ for Fis1 siRNA vs Fis1 siRNA+LNAME. For high glucose conditions, $\mathbf{B}, \mathrm{n}=9$, overall $\mathrm{P}=0.04 ; \mathrm{P}=0.01$ for scrambled siRNA vs Fis 1 siRNA; $\mathrm{P}=0.03$ for Fis 1 siRNA vs control siRNA+L-NAME and $\mathrm{P}=0.047$ for Fis 1 siRNA vs Fis1 siRNA+L-NAME. The box represents 25 and 75 percentiles with the horizontal line representing the median.

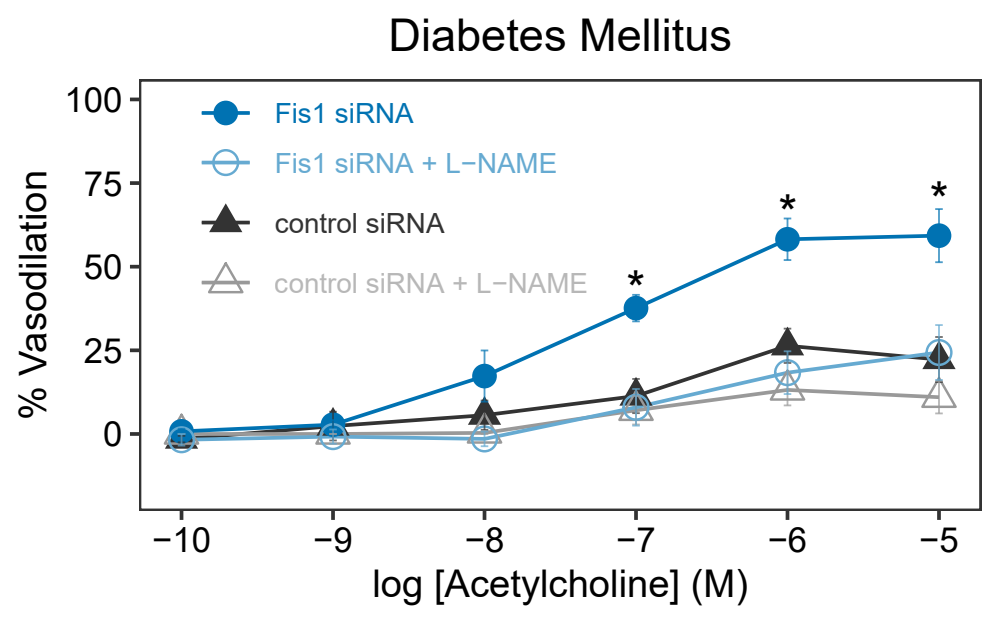

Figure 3: Suppression of Fis1 expression in T2DM human vessels reverses impaired endothelium-dependent vasodilation. Human resistance vessels isolated from T2DM were treated with Fis 1 siRNA and vasodilation measured by videomicroscopy, $n=6$, $\mathrm{P}=0.002$ overall. Treatment with $100 \mu \mathrm{M}$ L-NAME blocked this effect, $\mathrm{P}<0.0004$ for Fis1 siRNA vs Fis1 siRNA+L-NAME. ${ }^{*} \mathrm{P}<0.0005$ for vs. all other exposures at the indicated acetylcholine doses. Uncertainties are standard error of the mean.

Suppressing Fis 1 improves NO bioavailability in HMEC-1 cells

To further examine the effects of Fis1 on endothelial cells, we turned to immortalized human microvascular endothelial cells (HMEC-1) and validated this model by confirming their responsiveness to the calcium ionophore, A23187, that increases eNOS activation and NO bioavailability (Supplemental Figure 6A, $\mathrm{N}=6, \mathrm{P}=0.03 ; \mathbf{6 B}$, $\mathrm{N}=4, \mathrm{P}=0.02$ ). Consistent with human arterioles, transfection of HMEC-1 cells with Fis 1 siRNA also significantly increased NO bioavailability as measured by DAF-2 DA signal upon A23187 stimulation (Supplemental Figure 7, $\mathrm{N}=7, \mathrm{P}<0.0001$ ). To evaluate the specificity of Fis1 knockdown, we measured protein expression levels involved in mitochondrial function (CYT C, POLG, NDUF88, AMPK), fission (DRP1, MFF), fusion (MFN1, MFN2, OPA1), and autophagy (GABARAP, P62) and found no significant change even upon incubation under low, normal and high glucose conditions (Supplemental Figure 8). These data suggest that Fis1 siRNA treatment did not grossly affect mitochondrial homeostasis and HMEC-1 may be suitable for further investigation of the Fis1 effect.

Fis 1 knockdown improves endothelial cell layer integrity with no impact on bioenergetics

Endothelial cells provide an important barrier in human arterioles and to assess a potential role of Fis 1 in this function, we measured electrical resistance of HMEC-1 
monolayer under normal, low, and high glucose conditions. Under normal glucose conditions, Fis1 siRNA treatment decreased electrical resistance slightly suggesting a modest role for Fis1 in maintaining tight junction integrity under normal glucose conditions (Figure 4). Under both low and high glucose conditions, HMEC-1 electrical resistance significantly decreased as expected from these conditions which are known to impair vascular barrier function. Fis1 knockdown significantly increased resistance under both low and high glucose conditions but not to normal glucose levels. Since the removal of Fis1 improves monolayer barrier function only under abnormal glucose exposure, these data support that Fis 1 overexpression, as we observe in human arterioles, may contribute to impairing barrier function.

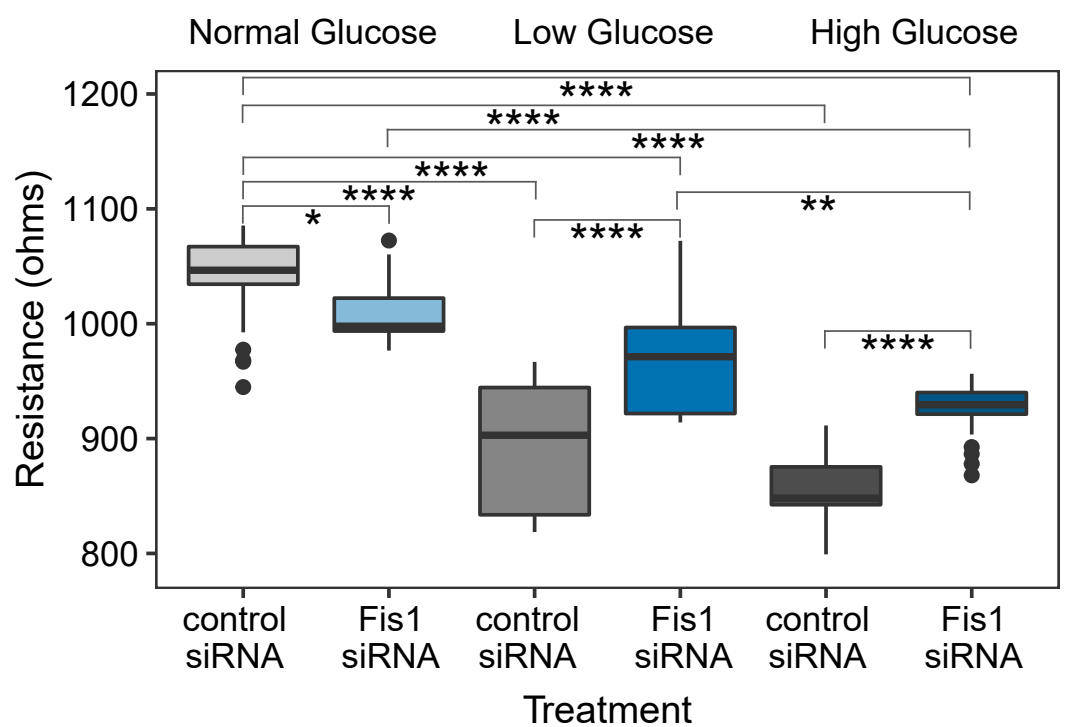

Figure 4: Suppression of Fis1 expression under low (2.5mM) and high (33 $\mathrm{mM})$ glucose conditions improves steady-state junction stability in endothelial cell monolayers. Comparison of electric cell-substrate impedance sensing measurements (ECIS) in human microvascular endothelial cell (HMEC-1) transfected with Fis1 siRNA and control siRNA between normal $(5 \mathrm{mM})$, low $(2.5 \mathrm{mM})$, and high $(33 \mathrm{mM})$ glucose conditions. Box represents 25 th to 75 th percentiles; horizontal line represents the median. SANOVA followed by Tukey's multiple comparison test, $\mathrm{n}=4$ for each treatment, $\mathrm{P}<0.001$ overall for both high and low glucose studies. ${ }^{*}-\mathrm{P}<0.05,{ }^{*} * \mathrm{P}<0.01,{ }^{*} * \mathrm{P}<0.001$, $* * * * \mathrm{P}<0.0001$.

The Fis 1 dependent changes in barrier function were unexpected and could arise from changes in cellular bioenergetics required for proper barrier function. To address this, we used extracellular flux measurements of glycolysis and oxidative phosphorylation in HMEC-1 cells at normal, low, and high glucose conditions upon Fis 1 knockdown (Figure 5). Neither extracellular acidification nor oxidative phosphorylation rates were impacted by glucose conditions as expected. Knockdown of Fis 1 also had no effect suggesting that inhibition of Fis 1 does not significantly impact cellular bioenergetics. 

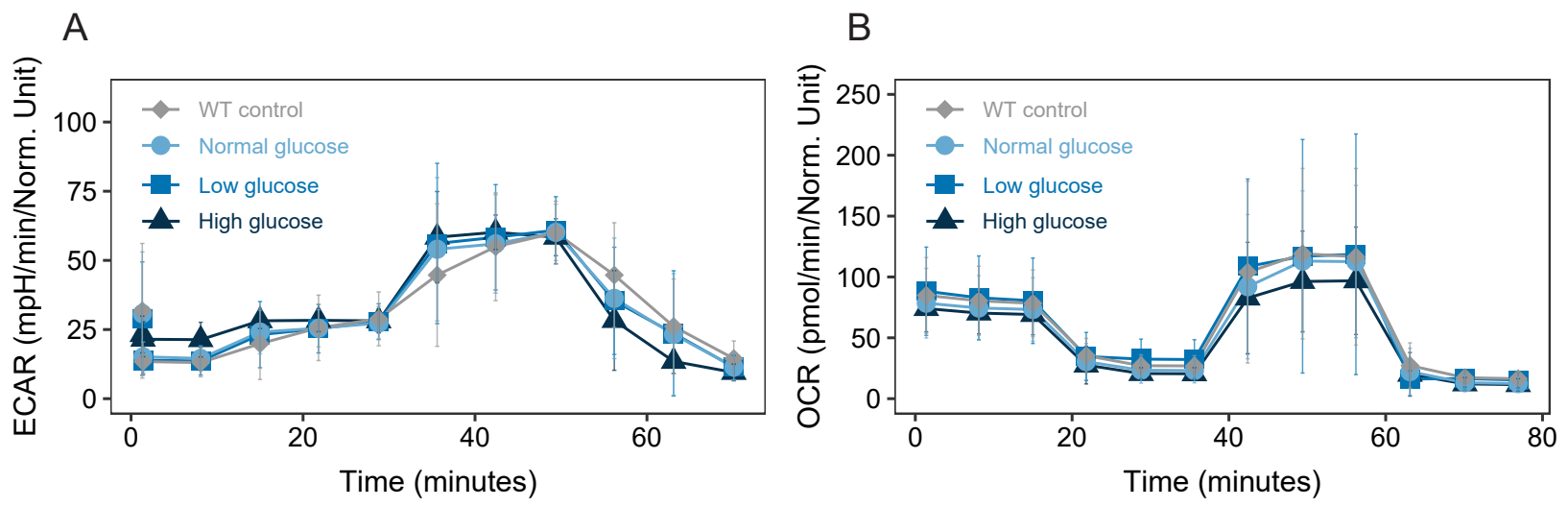

Figure 5: Suppression of Fis1 expression under low (2.5mM) and high (33mM) glucose conditions do not alter cellular bioenergetics. Measures of extracellular acidification rate (ECAR, $n=5)(A)$ and oxygen consumption rate $(\mathrm{OCR}, \mathrm{n}=5)(\mathbf{B})$ in human microvascular endothelial cells (HMEC-1) transfected with Fis1 siRNA and control siRNA pre-incubated with normal (5mM for 2 hours), low (2.5 $\mathrm{mM}$ for 2 hours) and high (33 $\mathrm{mM}$ for 6 hours) glucose conditions. Wild type (WT) cells represent HMEC-1 cells not transfected and grown under normal glucose conditions. ECAR and OCR were measured under basal conditions followed by the sequential addition of the indicated compounds. No statistically significant differences were present between the treatments.

\section{Determination of the affinity of pep213 for Fis 1:}

The ability of reduced Fis1 levels to improve aspects of endothelial cell function in vitro and ex vivo without impairing cellular bioenergetics suggested pharmacological inhibition of Fis1. Several potential Fis1 binding peptides were previously identified by phage display screening against a truncated version of the protein (23). We tested a subset of these peptides for binding to Fis 1 and found high $\mu \mathrm{M}$ affinity not suitable for testing in human arterioles (data not shown). From these data and computational modeling, we designed a novel peptide, pep213, that we reasoned might have higher affinity to Fis1. To evaluate this, we titrated increasing amount of the peptide into an NMR sample of Fis1 uniformly labeled with the stable isotope ${ }^{15} \mathrm{~N}$. A superposition of the resulting ${ }^{1} \mathrm{H}^{15} \mathrm{~N}$ correlation spectra show many signals change upon addition of pep213 indicating binding (Figure 6A). The resulting data can be globally fit to a single site binding model and gave an apparent $K_{D}=7 \pm 2 \mu \mathrm{M}$ (Figure 6B). This affinity was confirmed by separate tryptophan fluorescence experiments in which changes in the tryptophan emission spectra upon addition of pep213 were fit to a binding isotherm to give a similar apparent $K_{D}=3.3 \pm 0.1 \mu \mathrm{M}$ (Figure 6C). Another peptide (pep213scrambled), consisting of the same amino acid composition as pep213 but in random order, showed no chemical shift perturbations upon addition to ${ }^{15} \mathrm{~N}$-Fis 1 at $2 \mathrm{mM}$ indicating the Fis 1-pep213 interaction is specific (Figure 6D). 


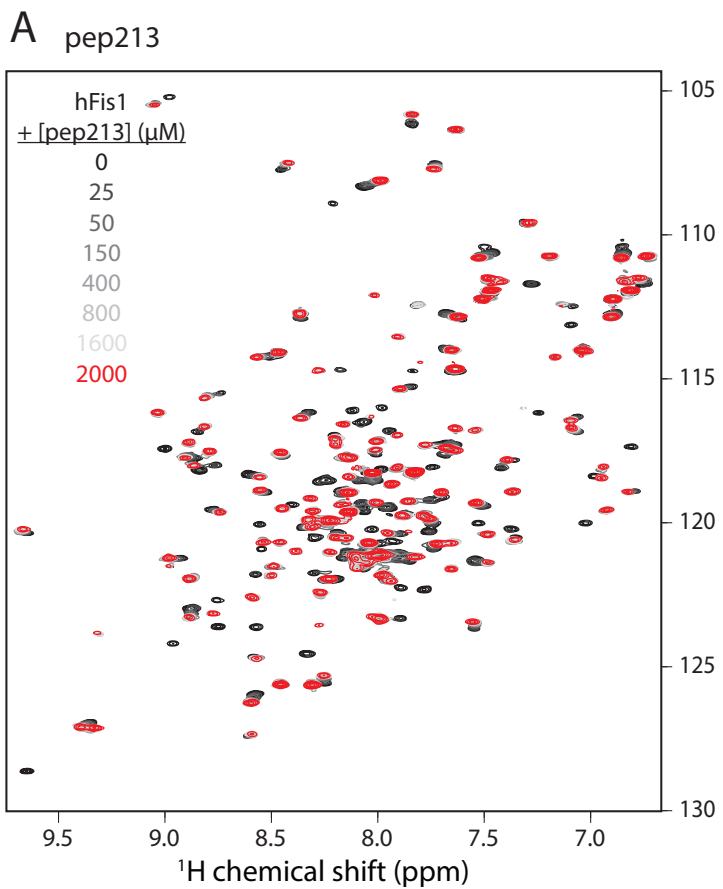

B
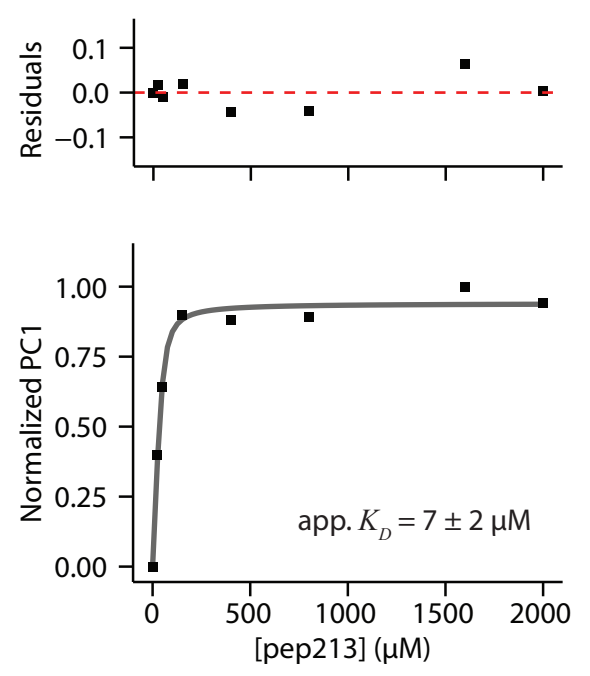

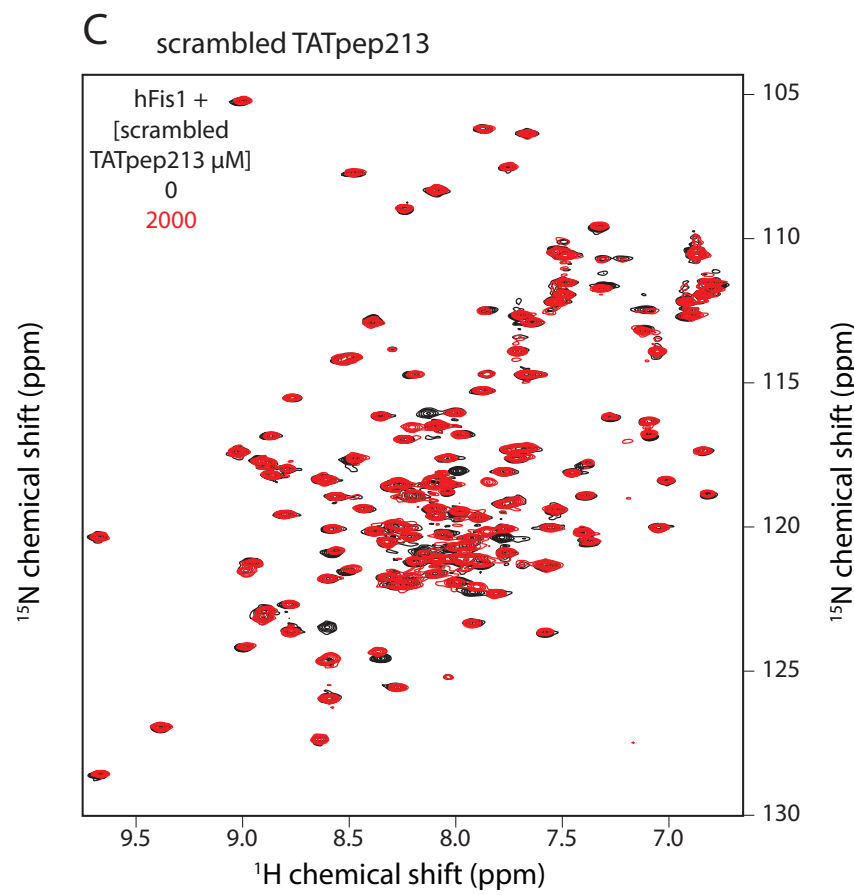

D
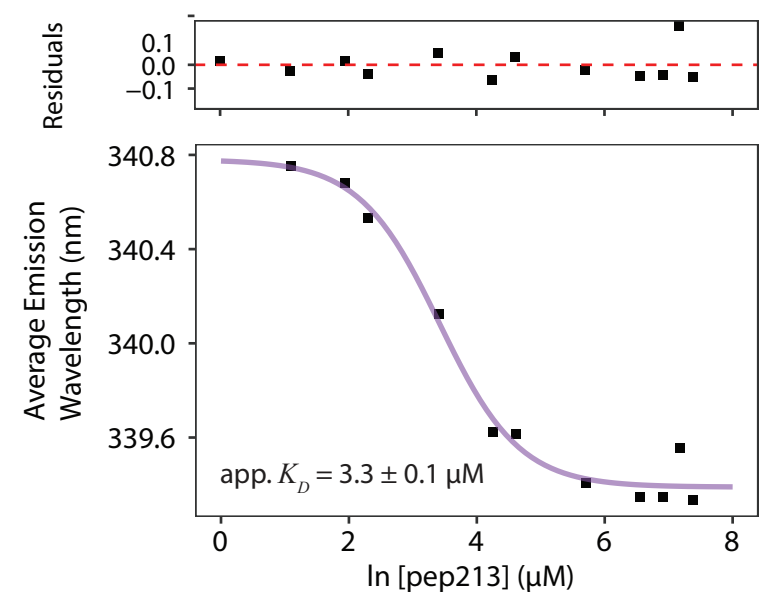

Figure 6: Novel peptide pep213 binding to Fis1. (A) ${ }^{1} \mathrm{H},{ }^{15} \mathrm{~N}$ HSQC spectral overlay of a $50 \mu \mathrm{M}{ }^{15} \mathrm{~N}$-Fis 1 sample titrated with increasing amounts $(0-2000 \mu \mathrm{M})$ of unlabeled p213 demonstrating significant changes in chemical shifts indicating affinity of p213 for Fis 1. (B) Affinity determination for pep213 binding to Fis1 from NMR data in (A) by applying TREND analysis to all spectra in the titration series and fitting the normalized principal component 1 values (PC1) to a ligand depletion model to determine an apparent $K_{D}=7 \pm 2 \mu \mathrm{M}$. (C) Affinity determination by intrinsic tryptophan fluorescence. $10 \mu \mathrm{M}$ Fis1 was titrated with increasing amounts $(0-1000 \mu \mathrm{M})$ of pep213. The average emission wavelength was fit to determine an apparent $K_{D}=3.3 \pm 0.1 \mu \mathrm{M}$. Residuals to the fits in panels B and C are shown in the top of each panel. (D) ${ }^{1} \mathrm{H},{ }^{15} \mathrm{~N}$ HSQC spectral overlay of a $50 \mu \mathrm{M}{ }^{15} \mathrm{~N}$-Fis1 sample titrated with $2000 \mu \mathrm{M}$ of a pep213-scrambled 
peptide with identical amino acid composition as p213 demonstrating no significant chemical shift changes indicating no affinity for Fis1.

Pep213 improves endothelial-dependent vasodilation in human T2DM resistance vessels

To test whether Fis1 inhibition might improve vasodilation in human arterioles, we first incubated vessels from healthy subjects exposed to high glucose with TATpep213, a version of pep213 fused to the cell permeant TAT peptide. Consistent with the siRNA knockdown of Fis1, TATpep213reversed the poor vasodilatory response in healthy vessels induced by high glucose (Figure 7A, $N=6, P<0.0001$ overall). This response was specific to pep213 as incubation with TATpep213-scrambled(that does not engage Fis1) showed no improvement (Figure 7C). Treatment with L-NAME abolished the improvements indicating an NO-dependent effect on vasodilation. We next asked if pep213 might improve NO-dependent vasodilation in vessels from T2DM patients, and consistent with the siRNA knockdown of Fis1, pep213 reversed the poor vasodilatory response in diabetic vessels (Figure 7B, N=4, $<<0.05$ overall). Again, this effect was specific to Fis1 inhibition as the scrambled peptide showed no improvement (Figure 7D). No differences were seen in the papaverine responses (data not shown) in any of these studies suggesting TATpep213did not impact smooth muscle reactivity. These results support that inhibiting Fis1 activity improves vasodilatory function in diabetic vessels.

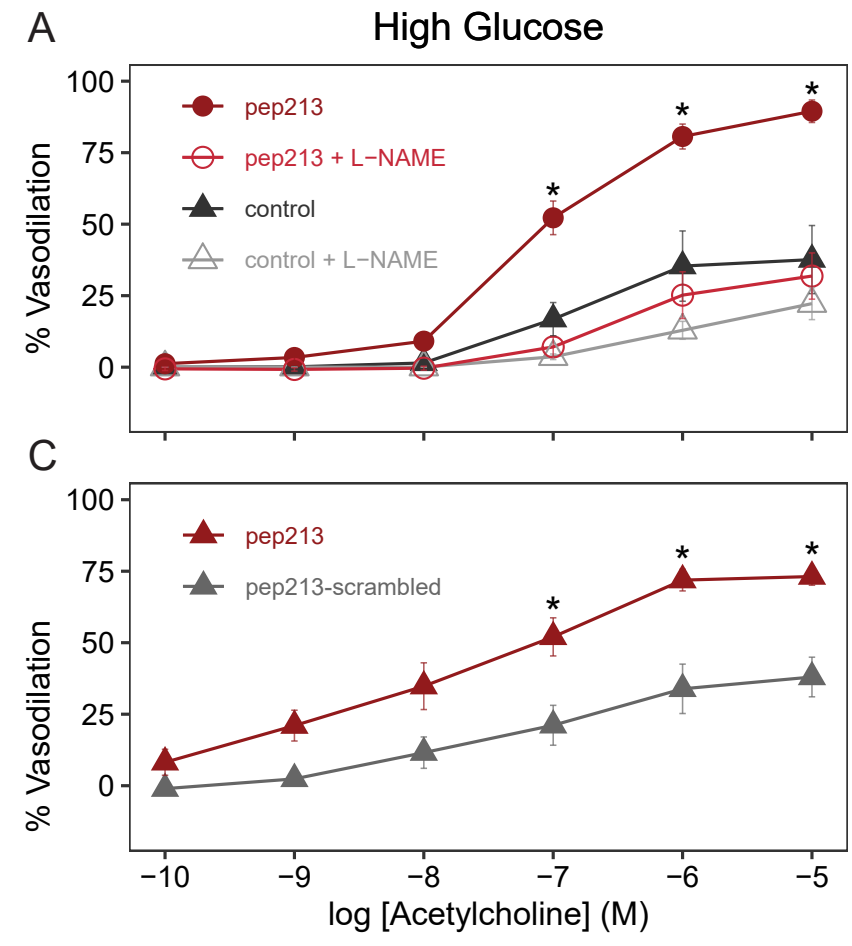

B Diabetes Mellitus

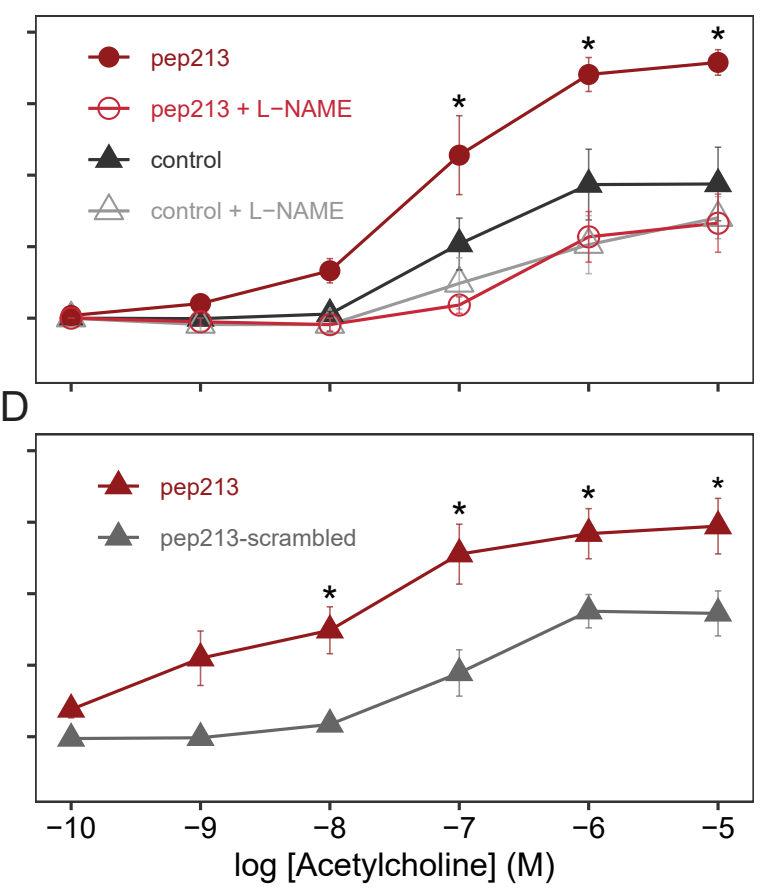

Figure 7: Pep213, but not the scrambled control, reverses impaired endothelium dependent vasodilation in human vessels from healthy and T2DM subjects. Human resistance vessels isolated from normal healthy $(\mathbf{A}, \mathbf{C})$ or T2DM (B,D) subjects were treated with a TAT fusion to the indicated peptide and vasodilation measured by videomicroscopy upon acetylcholine treatment. The impaired vasodilation caused by high 
glucose conditions $(\mathbf{A}, 33 \mathrm{mM})$ was reversed by treatment with $1 \mu \mathrm{M}$ of TATpep213 $(\mathrm{n}=5, \mathrm{P}<0.0001)$. L-NAME reversed all improvements with TATpep213 treatment $(\mathrm{P}<0.0001$ for pep 213 vs pep-213+L-NAME). $* \mathrm{P}<0.0001$ at the indicated acetylcholine concentration for pep213 vs. all other exposures). (B) The impaired endotheliumdependent vasodilation to acetylcholine in vessels from T2DM subjects was reversed by $1 \mu$ M TATpep213 $(\mathrm{P}<0.001$ overall, $* \mathrm{P}<0.05$ at the indicated acetylcholine concentrations for TATpep213 vs. all other exposures). (C) Acetylcholine induced endothelium-dependent vasodilation pre-exposed to six hours of high glucose $(33 \mathrm{mM})$ was reversed with $1 \mu \mathrm{M}$ TATpep213 compared to a scrambled peptide containing the same amino acids as pep213 attached to a TAT sequence. $(\mathrm{n}=5, \mathrm{P}<0.001$ overall, $* \mathrm{P}<0.05$ at indicated acetylcholine concentrations). (D) Similar results were seen with vessels from T2DM subjects ( $\mathrm{n}=4, \mathrm{P}<0.001$ overall, $* \mathrm{P}<0.05$ at the indicated doses).

\section{Discussion:}

These investigations reveal several novel findings. First, molecular inhibition of Fis1 expression reverses impairment of endothelium-dependent vasodilation in human vessels from T2DM patients. Additionally, molecular inhibition of Fis 1 blocks the unfavorable impacts of high- and low-glucose exposures on the ability of human microvascular endothelial cells to produce NO, vasodilate in an endothelium-dependent manner, and maintain the endothelial cell layer barrier function. These favorable effects occur without changes in endothelial cell metabolism or the expression of other proteins involved in mitochondrial fusion, fission, or autophagy. These findings suggest a critical mechanistic role for excessive Fis1 expression and activity in the impairment of endothelial function in the settings of both acute and chronically abnormal glucose levels. Additionally, these data support the concept that a pharmacological therapy targeting to Fis 1 holds promise for improving vascular health in patients with type 2 diabetes.

A strong rationale exists for exploring whether targeting mitochondrial proteins involved regulating the mitochondrial dynamics would lead to a favorable impact on human vascular endothelial function in patients with type 2 diabetes, or patients exposed to high glucose concentrations. Properly balanced mitochondrial networks are critical for maintaining normal mitochondrial function. Normal mitochondrial dynamics allow mitochondria to move to areas of increased metabolic demand, repair damaged mitochondria, maintain normal mitochondrial energetics, limit reactive oxygen species production, and isolate irreversibly damaged mitochondrial components for autophagy (24-28). The acute and chronic exposure to excessive nutrients, such as elevated glucose or free fatty acids, stimulates the mitochondrial network to undergo fission in multiple cells types, including human endothelial cells, leading to excessive mitochondrial ROS production $(1,28-30)$. Seminal work in cultured endothelial cells demonstrates that high glucose driven excessive mitochondrial ROS production that leads both acute and chronic impairment endothelial cell function through cell signaling and epigenomic pathways $(3$, 31). We have also previously demonstrated that reducing mitochondrial superoxide levels in resistance arteries from patients with type 2 diabetes reverses impaired endotheliumdependent vasodilation in human small resistance vessels suggesting an important connection between mitochondrial ROS and dynamics (2). 
These data suggest that targeting mitochondrial dynamics proteins to reduce excessive mitochondrial fragmentation could benefit vascular health in vessels acutely or chronically exposed to abnormal glucose levels. While there are multiple proteins involved in mitochondrial fusion and fission processes, Fis1, located on the outer mitochondrial membrane and a docking protein for Drp1, has been repeatedly shown to be over-expressed in the setting of diabetes, acute high glucose exposure, or acute exposure to excessive free fatty acids in multiple cell types $(1,32-35)$. While Fis 1 is not necessary for all fission(36), Fis1-Drp1 mediated fission appears to be preferred under conditions of cellular stress such as hypoxia and excessive glucose exposure (9-12). Prior work in the human vasculature demonstrate Fis1 is overexpressed in endothelial cells from individuals with type 2 diabetes (1). In addition, high glucose exposure leads to over-expression of Fis1 in cultured human aortic endothelial cells, and molecular silencing of Fis1 or Drp1 expression in human aortic endothelial cells exposed to high glucose results in an increase in phosphorylation of eNOS at its Ser1177 activation site (1). Additionally, we previously showed that clinically relevant levels of low glucose exposure leads to mitochondrial fragmentation in human endothelial cells and excessive mtROS production that can be reversed by suppressing mitochondrial fission protein activity $(8)$. Our new data significantly extends the translational impact of prior work by showing that molecular inhibition of Fis1 reverses impaired endothelium-dependent vasodilation in intact human resistance arteries, increases NO bioavailability in these vessels, and protects endothelial cell layer integrity in the setting of abnormal glucose levels. In addition, we determined that targeting Fis 1 does not impact endothelial cell mitochondrial metabolism or the expression of other mitochondrial proteins involved with dynamics or autophagy, reassuring observations when considering potential offtarget effects of therapeutically targeting Fis 1.

We found pep213, designed to bind Fis 1 at a key interaction surface, both increased NO bioavailability in cultured endothelial cells and reversed both type 2 diabetes and high glucose-induced impairment of endothelium-dependent vasodilation. In concert with our molecular results, our data using the novel pep213 peptide support that direct pharmacological inhibition of Fis1 has favorable effects on the diabetic vasculature. Interestingly, several medications given as treatment of type 2 diabetes, two of which reduce cardiovascular risk in T2DM, also inhibit mitochondrial fission as an "off-target" effect by reducing Fis1 and/or Drp1 expression. Empagliflozin, an SGLT2 inhibitor with favorable effects on cardiovascular risk, mortality, and microvascular renal disease in T2DM patients $(37,38)$, used in rat model of T2DM (OLEFT), reduced Fis1 over-expression, reduced mitochondrial fission, and up-regulated mitochondrial superoxide dismutase, SOD2, in cardiac myocytes (39). Metformin, a long-time first-line agent for T2DM glucose control with favorable cardiovascular effects, reduces atherosclerotic formation in ApoE knockout mice by suppressing Drp1-mediated fission (40). The dipeptidyl peptidase 4 inhibitor, vildagliptin, from a class of medications known to increase NO production, reduces expression of Fis1 and Drp1, reduces Drp1 translocation from the cytosol, and reduces mitochondrial fission and ROS production, while increasing NO production in the aortic endothelium of diabetic mice (41). Interpreted within the framework of our presented data, these common anti-diabetes medications may have ameliorative vascular effects in T2DM in part based on off-target effects on the expression and/or interaction of Fis1 and Drp1. Whether these 
improvements are due to improved glycemic control or direct inhibition of Fis1 or Drp1 interaction merits future study.

Our study has some limitations. First, we focused primarily on the Fis1-Drp1 axis in regulating the vascular effects of T2DM and abnormal glucose exposures due to prior data supporting this axis as being most relevant in the setting of hyperglycemia and other pathological stimuli and our prior work supporting Fis 1's pathophysiological role in the diabetic endothelium $(1,9-12)$. Whether or not blocking Fis1 impairs interactions with Drp1 or with other mitochondrial docking proteins (Mff, MiD49/51) is unknown and merits future investigation. We did also not focus on mitochondrial fusion proteins (e.g. OPA1, Mfn1, Mfn2). Emerging data suggest Mfn2 expression downregulated in the tissues of patients with T2DM and Fis1 may also drive fission by inhibiting the GTPase activity of fusion proteins which may further reduce NO bioavailability $(1,42-44)$. The fusion pathway's role in the regulation of human vascular endothelial function and its potential interaction with Fis 1 merits also merits additional investigation. We did not perform osmotic controls with our experiments. However, we have done these controls with similar intact vessel and endothelial cell experiments on mitochondrial regulation of vascular function in our prior work and have never observed osmotic differences to influence our results $(4,8)$. Balanced against these limitations is the novelty of our findings in highly disease-relevant tissues from humans, and the development of novel intervention to blunt and reverse the adverse effects of diabetes on the human vasculature targeting the mitochondrial fission machinery. Future studies will focus on developing a higher affinity inhibitor of Fis1 with better pharmacological properties that will allow translation into the clinic.

Acknowledgement: We gratefully acknowledge Ms. Cathy Paddock and Dr. Peter Newman for training and access to the ECIS instrument for TEER measurements.

Sources of Funding: Dr. Puppala received support from GM089586. Dr. Hofeld is supported by R38HL143561. Dr. Hill receives support from HL128240 and GM067180. Dr. Widlansky receives support from HL128240, HL125409, HL144098, R38HL143561, and R61AT010680. Mr. Trykall was supported by T35HL0724485 and a participant the Medical College of Wisconsin's Molecular and Cellular Research Pathway.

Disclosures: Drs. Hill and Widlansky receive significant support from research grant HL128240 directly relevant to this manuscript. The authors declare they have no affiliations with or involvement in any organization or entity with any financial interest, or non-financial interest in the subject matter or materials discussed in this manuscript.

\section{Materials and Methods}

Subject Recruitment and Screening: We recruited 67 individuals with T2DM and healthy individuals (ages 21-75) without cardiovascular risk factors as previously described $(2,13)$. Individuals with T2DM were either on medications to treat T2DM and/or met criteria for the diagnosis of T2DM American Diabetes Association (14). Study methods were reviewed and approved by the Institutional Research Board of the Medical College of Wisconsin and all subjects signed a written informed consent form prior to proceeding with any study activities. All subjects were screened to assure they met study inclusion criteria. In all subjects, height and weight were measured and heart rates and blood pressures measured in triplicate. Subjects with or without T2DM were 
excluded if they had any of the following: known atherosclerotic disease (coronary artery disease, peripheral vascular disease, history of stroke or myocardial infarction), chronic liver disease, elevated plasma creatinine ( $>1.5 \mathrm{mg} / \mathrm{dl}$ in men, $>1.4 \mathrm{mg} / \mathrm{dl}$ in women), a diagnosis of cancer in remission less than a year, regularly taking a blood thinner or antiplatelet agent other than aspirin, or smoking cigarettes within a year of enrollment. Individuals without T2DM were also excluded if they had an LDL cholesterol $\geq 160$ $\mathrm{mg} / \mathrm{dl}$ or hypertension (blood pressure $\geq 140 / 90 \mathrm{mmHg}$ ) or if they were on medications to treat either of these entities. Vessels for the studies comparing the impact of TATpep213 and scrambled control peptides on endothelium dependent vasodilation ( $\mathrm{N}=5$ non-T2DM, $\mathrm{N}=4$ T2DM) were obtained from discarded subcutaneous adipose tissue under a separate protocol reviewed and approved by the Medical College of Wisconsin's Institutional Research Board.

Human Resistance Artery Acquisition: Human resistance arteries from adipose samples were obtained from the upper outer quadrant of the gluteal adipose pad as previously described $(2,8,13,15,16)$. Briefly, following sterilization and anesthesia with $1 \%$ lidocaine, a $1-1.5 \mathrm{~cm}$ incision is made in the upper outer quadrant of the gluteal adipose pad. An approximately $8 \mathrm{~cm}^{3}$ volume of adipose tissue was removed by sharp dissection. After achieving hemostasis, the wounds were closed with 1-2 absorbable deep dermal sutures and the epidermal layer closed using either Dermabond or steristrips.

Cell Culture: Immortalized human microvascular endothelial cells (HMEC-1) purchased from ATCC (Manassas, VA) were cultured in antibiotic-free MCDB131 (Life Technologies, Carlsbad, CA) supplemented with $10 \mathrm{mM}$ Glutamine (Thermo Fisher Scientific, Waltham, MA), 10\% FBS (Sigma-Aldrich, St. Louis, MO), 10 ng/mL Human EGF (Thermo Fisher Scientific), and $1 \mu \mathrm{g} / \mathrm{mL}$ Hydrocortisone (Sigma-Aldrich). For assays involving high glucose $(33 \mathrm{mM})$, normal glucose $(5 \mathrm{mM})$, or low glucose $(2.5$ $\mathrm{mM}$ ) conditions, antibiotic-free MCDB131 was supplemented with the above mentioned and glucose levels were adjusted by adding glucose and sterile PBS. Human dermal microvascular endothelial cells (HMEC) were purchased from Lonza (Basel, Switzerland) and supplemented with Microvascular Endothelial Cell Growth Medium-2 Bullet Kit (Lonza).

\section{Transfection Protocols for Cultured Endothelial Cells and Human Vessels}

Transfection of Fis1 and Drp1 siRNA and Scrambled Control into Cultured Cells Constructs for Fis1 and Drp1 siRNAs were acquired from Origene (Rockville, MD, sequences included in the supplemental material). Lipofectamine RNAi Max (Thermo Fisher Scientific) vehicle was added to $20 \mathrm{nM}$ of RNAi constructs in Opti-MEM (Life Technologies). The mixture was diluted in culture media and incubated with HMEC-1 cells for four hours prior replacement with normal culture media. Cells were subsequently incubated for 24 hours before treatment and analyses. Following incubation, cells treated exposed to either high glucose for 6 hours or low glucose for 2 hours prior to measuring NO production, bioenergetics, protein expression, and endothelial layer integrity.

Transfection of Fis1 and Drp1 siRNA and Scrambled Controls in Human $\underline{\text { Resistance Arteries }}$ 
Transfection of human resistance arteries with siRNA constructs was performed as previously described $(8,13)$. Briefly, resistance arteries dissected from adipose tissue were suspended in a culture myograph chamber (204 CM, DMT, Ann Arbor). One end of the vessel was sutured onto a glass pipette within a micro-organ chamber. Prior to suturing the second end of the arteriole to a glass pipette, Fis1 siRNA, Drp1 siRNA (20 $\mathrm{nM}$ in optiMEM using Lipofectamine RNAiMax, Invitrogen) or control scrambled siRNA $(20 \mathrm{nM})$ were gently injected, into the vessel lumen. The loose end of the vessel is then tied onto another glass pipette, and the vessel is suspended in the chamber and placed on a myograph stage bathed in physiological buffer at $37^{\circ} \mathrm{C}$ and pressurized to 60 $\mathrm{mmHg}$. Following 4-6 hours of incubation, the siRNA is slowly washed out of the lumen over 24 hours at a low shear rate $\left(<5 \mathrm{dyn} / \mathrm{cm}^{2}\right)$.

Measurement of Endothelium-Dependent Vasoactivity: Transfected resistance arteries from healthy individuals were exposed to either normal glucose conditions ( 5 $\mathrm{mM}), 2$ hours of low glucose $(2.5 \mathrm{mM})$ or 6 hours of high glucose $(33 \mathrm{mM})$. All studies in vessels from patients with T2DM were performed at $5 \mathrm{mM}$ glucose. Vessels were preconstricted approximately 50\% of resting diameter with endothelin-1 (Sigma Aldrich, USA). The constricted arterioles are then exposed to acetylcholine (acetylcholine) at serially increasing doses from $10^{-10}$ to $10^{-5} \mathrm{M}$, and changes in vessel diameter were measured using digital calipers and videomicroscopy. Following the $10^{-5} \mathrm{M}$ dose, vessels were exposed to $200 \mu \mathrm{M}$ papaverine to test the smooth muscle reactivity. Following a 30minute washout period, the same arterioles were re-constricted and incubated with $N_{\omega^{-}}$ Nitro-L-arginine methyl ester (L-NAME, $100 \mu \mathrm{M})$, a direct inhibitor of nitric oxide synthase, for 30 minutes and vasodilation subsequently was remeasured following the exposure to acetylcholine $10^{-10}$ to $10^{-5} \mathrm{M}$.

\section{Measurements of Nitric Oxide (NO) Bioavailability:}

Cells in Culture: To measure nitric oxide (NO) bioavailability in HMEC-1 cells and arterioles, a fluorescent NO marker, 4,5-diaminofluorescein diacetate (DAF-2 DA from Cayman Chemical) was used. In a dark room, cells were incubated with or without $100 \mu \mathrm{M}$ L-NAME for 2 hours followed by 15 minutes of incubation with $5 \mu \mathrm{M}$ DAF-2 $\mathrm{DA}$ at $37^{\circ} \mathrm{C}$. Fluorescence intensity was measured using a SPECTRAFluor Plus plate reader (Tecan, Morrisville, NC) using excitation and emission wavelengths of $485 \mathrm{~nm}$ and $535 \mathrm{~nm}$, respectively.

Human Resistance Arteries: Two vessels were obtained from a subset of healthy subjects and each vessel was cut in half to allow for four experimental conditions which included transfection with Fis1 siRNA or Drp1 siRNA with and without concomitant exposure to L-NAME for 30 minutes and transfection with scrambled siRNA control with and without concomitant exposure to L-NAME at room temperature. acetylcholine $10^{-5} \mathrm{M}$ and DAF-2 DA (5 $\mu \mathrm{M}$ final concentration) were subsequently added to each vessel and incubated at $37^{\circ} \mathrm{C}$ for 30 minutes. The arterioles were then washed with PBS buffer, mounted on a slide, and imaged by fluorescence microscopy. Untreated and unstained arterioles were used as controls for fluorescence background interference. Both treated and control vessels were measured using the same gain settings and the results were analyzed using Meta Morph 7.8 software (Universal imaging, West Chester, PA).

\section{Measurement of Endothelial Layer Integrity:}


Human microvascular endothelial (HMEC-1) cells were seeded at 40,000 cells/ well and grown on gold electrode array plate (8W10E+, Applied Biophysics Inc.) until 50\% confluent. The cells were pre-transfected with Fis-1 siRNA (20 nM) or scrambled siRNA $(20 \mathrm{nM})$. On the days of experiments, the transfected cells were exposed to different glucose conditions: high glucose $(33 \mathrm{mM})$ for 6 hours, normal glucose $(5 \mathrm{mM})$ for 2 hours and low glucose $(2.5 \mathrm{mM})$ for 2 hours. The integrity of the monolayers were checked at $64,000 \mathrm{~Hz}$ with less than $10 \mathrm{nF}$ capacitance. The cells were then subjected to Electric Cell-substrate Impedance Sensing (ECIS) functional assay, and monolayer transepithelial electrical resistance (TEER) was measured in real time using an ECIS ZTheta Instrument (Applied Biophysics Inc., Troy, NY). Resistance was measured over the following frequencies, with $4,000 \mathrm{~Hz}$ as the standard: $125 \mathrm{~Hz}, 250 \mathrm{~Hz}, 500 \mathrm{~Hz}, 1,000$ $\mathrm{Hz}, 2,000 \mathrm{~Hz}, 4,000 \mathrm{~Hz}, 8,000 \mathrm{~Hz}, 16,000 \mathrm{~Hz}, 32,000 \mathrm{~Hz}$, and 64,000 Hz.

\section{Mitochondrial Bioenergetic Measurements:}

The XFe96 Analyzer (Seahorse Biosciences, North Billerica, MA, USA) was used to measure mitochondrial bioenergetics by measuring the oxygen consumption rate (OCR) and extracellular acidification rate (ECAR). HMEC-1 cells were seeded $(20,000$ cells/well) onto a 96-well Seahorse microplate 4-6 hours before treatment to let the cells adhere to the plate. Cells were pre-transfected with siRNA targeting Fis 1 and siRNA scrambled control. The medium was aspirated and replaced with either high glucose (33 $\mathrm{mM})$ for 6 hours or normal $(5 \mathrm{mM})$ or low $(2.5 \mathrm{mM})$ glucose for 2 hours. The high, normal, or low glucose medium was removed and replaced with XF Base Medium Minimal Dulbecco's modified Eagle's medium (DMEM, pH 7.40) supplemented with 600 $\mu \mathrm{L}$ glucose $45 \%, 1.5 \mathrm{~mL}$ L-Glutamine $(200 \mathrm{mM})$, and $1.5 \mathrm{~mL}$ sodium pyruvate $(100$ $\mathrm{mM}$ ) with the final glucose concentration the same as the initial medium concentration. Cells were then incubated in a $\mathrm{CO}_{2}$-free incubator at $37^{\circ} \mathrm{C}$ for 1 hour for temperature and $\mathrm{pH}$ calibration. During mitochondrial stress tests, the following drugs were sequentially injected in increments of $25 \mu \mathrm{L}$ : Oligomycin A ( $2.5 \mu \mathrm{M}$ final well concentration), FCCP (carbonyl cyanide 4-(trifluoromethoxy) phenylhydrazone, $1 \mu \mathrm{M}$ final well concentration), and rotenone $\&$ antimycin A ( $1 \mu \mathrm{M}$ final well concentration each). During glycolytic stress tests the following substances were sequentially injected in increments of $25 \mu \mathrm{L}$ : glucose (10 mM final well concentration), oligomycin A $(2.5 \mu \mathrm{M}$ final well concentration), and 2-deoxyglucose (50 $\mathrm{mM}$ final well concentration).

Following these measurements, cells were fixed with 4\% paraformaldehyde for 15 minutes and stained with DAPI (4',6-diamidino-2-phenylindole) at 1:500 of $1.5 \mu \mathrm{g} / \mathrm{mL}$ for 24 hours. Cell counts were obtained using automated cell counting on Cytation 5 cell imaging multi-mode reader (BioTek, Vermont, USA) with OCR and ECAR measurements then normalized to cell number.

\section{Measurement of Mitochondrial Proteins:}

HMEC-1 cells were grown on a six well plates at $0.3 \times 10^{6}$ cells/well and transfected with siFis 1 and siRNA. After transfecting for 4-6 hours, the medium was changed to normal cell culture medium and incubated overnight. Cells were treated either with high glucose $(33 \mathrm{mM})$ for 6 hours, or normal $(5 \mathrm{mM})$ or low $(2.5 \mathrm{mM})$ glucose for 2 hours. The plates were then washed $2 \mathrm{X}$ with cell wash buffer and $50 \mu 1$ RIPA lysis buffer (ProteinSimple, San Jose, CA) was added to each well. The cells were scraped gently on 
ice and transferred to a labelled tube. Lysates were centrifuged at $10,000 \mathrm{rpm}$ for 10 minutes and snap frozen in liquid nitrogen and kept at $-80^{\circ} \mathrm{C}$ overnight. The cell pellet and supernatant were thawed, vortexed briefly, and centrifuged at 10,000 rpm for 10 minutes. The supernatant was transferred to labelled tubes, protein concentration quantified by Bradford assay, and protein expression evaluated by automated capillary electrophoresis-based immunodetection via WES (ProteinSimple). Proteins were analyzed and detected with the $12-230 \mathrm{kDa}$ WES separation module and 25 capillary cartridges. Lysates were diluted in $0.1 \mathrm{X}$ sample buffer in a $4: 1$ ratio of sample to fluorescent master mix, then denatured at $95^{\circ} \mathrm{C}$ for 5 minutes. For immunodetection, blocking buffer, primary antibody, secondary antibody, chemiluminescent substrate, samples, a biotinylated size marker and wash buffer were loaded in designated wells on the supplied microplate. The plate was centrifuged $5 \mathrm{~min}$ at $1000 \mathrm{X} \mathrm{g}$ then loaded into WES. Protein detection used default separation parameters. Compass for SW software (version 3.1.7, ProteinSimple) analyzed data and integrated specific antibody peaks. Across samples, protein expression was normalized to total protein. Total protein was analyzed in an addition WES assay with biotin-based protein labeling. Total protein was also analyzed using Compass for SW software as the integral of the antibody peaks. Control carryover samples between plates were used to correct for inter-plate signal intensity variability. The average quotient of antibody peak integrals with sample over total protein was taken.

\section{NMR Titration Experiments:}

To determine peptide binding affinities for Fis1, NMR titration experiments were performed similarly to chemical fragment titrations as described (17). First, peptides were resuspended in Fis1 dialysate buffer (100 mM HEPES pH 7.4, $200 \mathrm{mM} \mathrm{NaCl}, 1 \mathrm{mM}$ DTT, $0.02 \% \mathrm{v} / \mathrm{v}$ sodium azide) to a final concentration of $6 \mathrm{mM}$. Then, $220 \mu \mathrm{L}$ of $50 \mu \mathrm{M}$ ${ }^{15} \mathrm{~N}-\mathrm{hFis} 1$ and increasing amounts of peptide $(0,25,50,150,400,800,1600$, and 2000 $\mu \mathrm{M}$ ) was prepared and loaded into $3 \mathrm{~mm}$ NMR tubes. For each sample, ${ }^{1} \mathrm{H},{ }^{15} \mathrm{~N}$ HSQC spectra were collected at $25^{\circ} \mathrm{C}$ on a Bruker Avance II $600 \mathrm{MHz}$ spectrometer equipped with a triple resonance z-axis gradient cryoprobe and SampleJet autosampler, which allowed automatic tuning, shimming, and data collection for each sample. ${ }^{1} \mathrm{H},{ }^{15} \mathrm{~N}$ HSQC experiments consisted of 8 scans with 1024 and 300 complex points in the ${ }^{1} \mathrm{H}$ and ${ }^{15} \mathrm{~N}$ dimensions, respectively. Spectra were processed with automated python scripts using NMRPipe and chemical shifts were measured using TitrView and CARA software (18, 19). Peptide binding affinity was determined by TREND analysis to each spectrum within the titration series $(20,21)$. TREND reveals the changes in data by performing principal component analysis, where each concentration point is treated as a unique data point; here, the input is each ${ }^{1} \mathrm{H},{ }^{15} \mathrm{~N}$ spectrum with increasing amounts of peptide. After performing principal component analysis, the principal component 1 (PC1) values are normalized to the highest PC1 value resulting in a range from 0 to 1 . Then, the normalized PC1 values are plotted against peptide concentration and fit to a ligand depletion function with protein concentration held constant (Eq. 1)

Equation 1: $\Delta=\frac{\mathrm{d}_{\max } *\left(\left(K_{d}+p+l\right)-\left(\left(K_{d}+p+l\right)^{2}-4 * p * l\right)^{0.5}\right)}{2 * p}$, where $\Delta=$ adjusted chemical shift change, $\mathrm{d}_{\max }=$ maximum chemical shift perturbation, $K_{d}=$ binding dissociation constant, $p=$ [protein], and $l=[$ ligand $]$. 
Spectral overlays were generated using XEASY software and Adobe Illustrator.

Synthesis of pep213:

Peptides pep213 (SHKHDPLPYPHFLL) and TAT-p213

(YGRKKRRQRRRGSGSGSSHKHDPLPYPHFLL) were all purchased from Genscript (Piscataway, NJ) with N-terminal acetylation and C-terminal amidation and $>95 \%$ purity by HPLC. The TAT-p213 fusion peptide included a GSGSGS linker between the TAT cell penetrating sequence (YGRKKRRQRRR) and pep213.

Intrinsic Tryptophan Fluorescence Spectroscopy to determine pep213 affinity for Fis 1:

Tryptophan fluorescence data was collected on a PTI Model \#814 fluorimeter using a $\lambda_{\text {ex }}$ of $295 \mathrm{~nm}$ and $\lambda_{\text {em }}$ of $300-400 \mathrm{~nm}$ within Starna Cells 3-Q-10 quartz fluorometer rectangular cell with a $10 \mathrm{~mm}$ pathlength and excitation/emission slit widths of 4/6 nm, respectively. A concentrated stock of peptide (pep213) was resuspended in final hFis1 dialysate buffer and a concentration series with the following points was prepared: $0,1,3,7,10,30,70,100,300,700$, and $1000 \mu \mathrm{M}$ peptide. For each titration point, tryptophan emission spectra were collected on samples excluding Fis 1 and then, 5 $\mu \mathrm{L}$ of $400 \mu \mathrm{M} \mathrm{hFis} 1$ was added to the sample for a final concentration of $10 \mu \mathrm{M} h \mathrm{hFis} 1$ and tryptophan emission spectra were recollected. To account for buffer and tyrosine fluorescence background from the peptide, difference emission spectra were generated by subtracting the background fluorescence intensities from spectra lacking Fis1. The average emission wavelength at each peptide concentration was calculated according to Equation 3 and plotted as a function of the natural log of peptide concentration, which was fit to a Boltzmann sigmoidal model (Equation 4) (22).

$$
\sum_{n=1}^{N} \operatorname{In} * \lambda_{n}
$$

Equation 3: $\langle\boldsymbol{\lambda}\rangle=\sum_{n=1}^{N} I_{n}$, where $\langle\boldsymbol{\lambda}\rangle=$ average emission wavelength, $I_{\mathrm{n}}=$ fluorescence intensity emitted at wavelength $\lambda_{n}$, and the summation calculated from $\lambda_{n}$ of 310 to $370 \mathrm{~nm}$.

Equation 4: $\lambda=A+\frac{(B-A)}{1+e^{\frac{K_{D-p}}{C}}}$, where $\lambda=$ average emission wavelength, $A=$ pretransition phase, $B=$ post-transition phase, $K_{D}=$ equilibrium dissociation constant, $p=$ natural $\log$ of the peptide concentration, and $c=$ slope of the transition phase.

\section{Impact of pep213 on Endothelial Cell NO Bioavailability and Endothelium-} Dependent Vasodilation: Vessels from a subset of subjects with and without T2DM were selected at random for these studies. Vessels from healthy subjects were pre-treated with HG (33 mM) for six hours and subsequently exposed to $1 \mu \mathrm{M}$ of pep213 attached to a TAT sequence to facilitate cellular uptake. Vessels from T2DM subjects were incubated under normal glucose conditions $(5 \mathrm{mM})$ and exposed to either $1 \mu \mathrm{M}$ of TATpep213. Endothelium-dependent vasodilation with increasing doses of acetylcholine, smooth muscle reactivity to papaverine, and eNOS-dependence of the vasodilatory response to acetylcholine using L-NAME were assessed as described previously $(2,4,8,13,15)$.

Statistical Analyses: 
Statistical analyses were performed with either GraphPad Prism V7.03 (GraphPad Prism version 8.0.0 for Windows, GraphPad Software, San Diego, California, USA) or SigmaPlot Version 12.5 (SysTATSoftware, San Jose, California, USA). P $<0.05$ were considered statistically significant. Box and whisker plots present data as median, $25^{\text {th }}$ $75^{\text {th }}$ percentile box, and whiskers extending to 1.5 times the inter-quartile range. All other data are presented as mean \pm SEM unless otherwise stated. All functional vessel data was analyzed by two-way ANOVA with post-hoc testing to determine the differences between groups (Dunnett's multiple comparison test) and dose-response (Tukey's multiple comparison tests). The TEER assay data and bioenergetics data were also analyzed by two-way ANOVA followed by post-hoc testing (Tukey's multiple comparison tests) to determine the differences between normal, low, and high glucose treatments. DAF-2 DA fluorescence intensity in human vessels, Fis1 knockdownefficiency in HMEC-1 cells, and measurements of mitochondrial protein amounts by Western blot were analyzed by one-way ANOVA followed by Tukey's multiple comparisons to assess differences between groups. DAF-2 DA fluorescence intensity in HMEC-1 cells transfected with siFis1 and scrambled siRNA were analyzed by two-way ANOVA followed by post-hoc testing (Tukey's multiple comparison tests). NO production and relative ratio of P-eNOS and $\beta$-actin in HMEC- 1 cells stimulated with A23187 were analyzed using paired Student's t-test.

\section{Supplementary Materials}

\section{Materials and Methods}

Supplemental Table 1: Demographic, clinical, and medication information and in vivo vascular function for 16 subjects with resistance arterioles that underwent Fis 1 siRNA transfection and studied for vasodilation in response to acetylcholine.

Supplemental Table 2: Demographic, clinical, and medication information and in vivo vascular function for 17 subjects with resistance arterioles that underwent Fis1 siRNA transfection and studied for biological NO production using DAF-2 DA fluorescence.

Supplemental Table 3: Demographic, clinical, and medication information and in vivo vascular function for 13 subjects with resistance arterioles that underwent Drp1 siRNA transfection and studied for vasodilation in response to acetylcholine.

Supplemental Table 4: Demographic, clinical, and medication information and in vivo vascular function for 16 subjects with resistance arterioles that underwent Drp1 siRNA transfection and studied for biological NO production using DAF-2 DA fluorescence.

Supplemental Table 5: Demographic, clinical, and medication information and in vivo vascular function for 10 subjects with resistance arterioles exposed to pep213 and studied for vasodilation in response to acetylcholine in Figure 7.

Supplemental Table 6: Demographic, clinical, and medication information and in vivo vascular function for 9 subjects with resistance arterioles exposed to TATpep213and scrambled peptide-TAT and studied for vasodilation in response to acetylcholine in Figure 8 . 
Supplemental Figure 1: Fis1 knockdown efficiency in human arterioles with siRNA treatment.

Supplemental Figure 2: Transfection with Drp1 siRNA protected against high glucoseinduced impairment in endothelium-dependent vasodilation.

Supplemental Figure 3: Knockdown of Drp1 expression with Drp1 siRNA protected against reductions in nitric oxide (NO) bioavailability in arterioles from healthy humans human arterioles exposed to high or low glucose conditions.

Supplemental Figure 4: Suppression of Drp1 expression in arterioles from humans with T2DM using Drp1 siRNA tended to reverse impaired endothelium-dependent vasodilation.

Supplemental Figure 5: Fis1 knockdown efficiency in HMEC-1 cells with siRNA treatment.

Supplemental Figure 6: Phosphorylation of eNOS at Ser1177 and NO production increased in HMEC-1 cells upon activation with $\mathrm{Ca}^{+2}$ ionophore A23187.

Supplemental Figure 7: Fis1 knockdown improved NO production in immortalized cultured human microvascular endothelial cells (HMEC-1) transfected with Fis1 siRNA.

Supplemental Figure 8: Molecular inhibition of Fis 1 under high glucose (33mM) and low glucose $(2.5 \mathrm{mM})$ conditions did not alter the expression of other mitochondrial proteins.

\section{References:}

1. S. M. Shenouda, M. E. Widlansky, K. Chen, G. Xu, M. Holbrook, C. E. Tabit, N. M. Hamburg, A. A. Frame, T. L. Caiano, M. A. Kluge, M. A. Duess, A. Levit, B. Kim, M. L. Hartman, L. Joseph, O. S. Shirihai, J. A. Vita, Altered mitochondrial dynamics contributes to endothelial dysfunction in diabetes mellitus. Circulation 124, 444-453 (2011).

2. T. J. Kizhakekuttu, J. Wang, K. Dharmashankar, R. Ying, D. D. Gutterman, J. A. Vita, M. E. Widlansky, Adverse alterations in mitochondrial function contribute to type 2 diabetes mellitus-related endothelial dysfunction in humans.

Arterioscler. Thromb. Vasc. Biol 32, 2531-2539 (2012).

3. A. El-Osta, D. Brasacchio, D. Yao, A. Pocai, P. L. Jones, R. G. Roeder, M. E. Cooper, M. Brownlee, Transient high glucose causes persistent epigenetic changes and altered gene expression during subsequent normoglycemia. J. Exp. Med 205, 2409-2417 (2008).

4. J. Wang, A. Alexanian, R. Ying, T. J. Kizhakekuttu, K. Dharmashankar, J. Vasquez-Vivar, D. D. Gutterman, M. E. Widlansky, Acute Exposure to Low Glucose Rapidly Induces Endothelial Dysfunction and Mitochondrial Oxidative Stress: Role for AMP Kinase. Arterioscler. Thromb. Vasc. Biol 32, 712-720 (2012).

5. S. Yusuf, G. Dagenais, J. Pogue, J. Bosch, P. Sleight, Vitamin E supplementation and cardiovascular events in high-risk patients. The Heart Outcomes Prevention Evaluation Study Investigators. N. Engl. J. Med 342, 154-160 (2000). 
6. B. J. Willcox, J. D. Curb, B. L. Rodriguez, Antioxidants in cardiovascular health and disease: key lessons from epidemiologic studies. Am J Cardiol 101, 75D-86D (2008).

7. M. E. Widlansky, R. B. Hill, Mitochondrial regulation of diabetic vascular disease: an emerging opportunity. Transl Res, (2018).

8. M. J. Tanner, J. Wang, R. Ying, T. B. Suboc, M. Malik, A. Couillard, A. Branum, V. Puppala, M. E. Widlansky, Dynamin-related protein 1 mediates low glucoseinduced endothelial dysfunction in human arterioles. Am J Physiol Heart Circ Physiol 312, H515-H527 (2017).

9. H. Kim, M. C. Scimia, D. Wilkinson, R. D. Trelles, M. R. Wood, D. Bowtell, A. Dillin, M. Mercola, Z. A. Ronai, Fine-tuning of Drp1/Fis 1 availability by AKAP121/Siah2 regulates mitochondrial adaptation to hypoxia. Mol. Cell 44, 532-544 (2011).

10. S. Kumari, L. Anderson, S. Farmer, S. L. Mehta, P. A. Li, Hyperglycemia alters mitochondrial fission and fusion proteins in mice subjected to cerebral ischemia and reperfusion. Transl. Stroke Res 3, 296-304 (2012).

11. L. Ciarlo, V. Manganelli, T. Garofalo, P. Matarrese, A. Tinari, R. Misasi, W. Malorni, M. Sorice, Association of fission proteins with mitochondrial raft-like domains. Cell Death. Differ 17, 1047-1058 (2010).

12. I. Kaddour-Djebbar, V. Choudhary, C. Brooks, T. Ghazaly, V. Lakshmikanthan, Z. Dong, M. V. Kumar, Specific mitochondrial calcium overload induces mitochondrial fission in prostate cancer cells. Int. J. Oncol 36, 1437-1444 (2010).

13. M. E. Widlansky, D. M. Jensen, J. Wang, Y. Liu, A. M. Geurts, A. J. Kriegel, P. Liu, R. Ying, G. Zhang, M. Casati, C. Chu, M. Malik, A. Branum, M. J. Tanner, S. Tyagi, K. Usa, M. Liang, miR-29 contributes to normal endothelial function and can restore it in cardiometabolic disorders. EMBO Mol Med, (2018).

14. A. American Diabetes, 2. Classification and Diagnosis of Diabetes: Standards of Medical Care in Diabetes-2019. Diabetes Care 42, S13-S28 (2019).

15. T. M. Suboc, K. Dharmashankar, J. Wang, R. Ying, A. Couillard, M. J. Tanner, M. E. Widlansky, Moderate Obesity and Endothelial Dysfunction in Humans: Influence of Gender and Systemic Inflammation. Physiol Rep 1, (2013).

16. A. Mohandas, T. B. Suboc, J. Wang, R. Ying, S. Tarima, K. Dharmashankar, M. Malik, M. E. Widlansky, Mineralocorticoid exposure and receptor activity modulate microvascular endothelial function in African Americans with and without hypertension. Vasc. Med, (2015).

17. J. M. Egner, D. R. Jensen, M. D. Olp, N. W. Kennedy, B. F. Volkman, F. C. Peterson, B. C. Smith, R. B. Hill, Development and Validation of 2D Difference Intensity Analysis for Chemical Library Screening by Protein-Detected NMR Spectroscopy. Chembiochem 19, 448-458 (2018).

18. F. Delaglio, S. Grzesiek, G. W. Vuister, G. Zhu, J. Pfeifer, A. Bax, NMRPipe: a multidimensional spectral processing system based on UNIX pipes. $J$ Biomol NMR 6, 277-293 (1995).

19. J. E. Masse, R. Keller, AutoLink: automated sequential resonance assignment of biopolymers from NMR data by relative-hypothesis-prioritization-based simulated logic. J Magn Reson 174, 133-151 (2005). 
20. J. Xu, S. R. Van Doren, Binding Isotherms and Time Courses Readily from Magnetic Resonance. Anal Chem 88, 8172-8178 (2016).

21. J. Xu, A. V. S. Sarma, Y. Wei, L. J. Beamer, S. R. Van Doren, Multiple LigandBound States of a Phosphohexomutase Revealed by Principal Component Analysis of NMR Peak Shifts. Sci Rep 7, 5343 (2017).

22. C. A. Royer, C. J. Mann, C. R. Matthews, Resolution of the fluorescence equilibrium unfolding profile of trp aporepressor using single tryptophan mutants. Protein Sci 2, 1844-1852 (1993).

23. M. N. Serasinghe, A. M. Seneviratne, A. V. Smrcka, Y. Yoon, Identification and characterization of unique proline-rich peptides binding to the mitochondrial fission protein hFis1. J Biol Chem 285, 620-630 (2010).

24. H. Chen, D. C. Chan, Mitochondrial dynamics--fusion, fission, movement, and mitophagy--in neurodegenerative diseases. Hum. Mol. Genet 18, R169-R176 (2009).

25. D. C. Chan, Fusion and fission: interlinked processes critical for mitochondrial health. Annu. Rev. Genet 46, 265-287 (2012).

26. S. A. Detmer, D. C. Chan, Functions and dysfunctions of mitochondrial dynamics. Nat Rev Mol Cell Biol 8, 870-879 (2007).

27. D. F. Suen, K. L. Norris, R. J. Youle, Mitochondrial dynamics and apoptosis. Genes Dev 22, 1577-1590 (2008).

28. T. Yu, J. L. Robotham, Y. Yoon, Increased production of reactive oxygen species in hyperglycemic conditions requires dynamic change of mitochondrial morphology. Proc. Natl. Acad. Sci. U. S. A 103, 2653-2658 (2006).

29. A. J. Molina, J. D. Wikstrom, L. Stiles, G. Las, H. Mohamed, A. Elorza, G. Walzer, G. Twig, S. Katz, B. E. Corkey, O. S. Shirihai, Mitochondrial networking protects beta-cells from nutrient-induced apoptosis. Diabetes 58, 2303-2315 (2009).

30. H. F. Jheng, P. J. Tsai, S. M. Guo, L. H. Kuo, C. S. Chang, I. J. Su, C. R. Chang, Y. S. Tsai, Mitochondrial fission contributes to mitochondrial dysfunction and insulin resistance in skeletal muscle. Mol Cell Biol 32, 309-319 (2012).

31. T. Nishikawa, D. Edelstein, X. L. Du, S. Yamagishi, T. Matsumura, Y. Kaneda, M. A. Yorek, D. Beebe, P. J. Oates, H. P. Hammes, I. Giardino, M. Brownlee, Normalizing mitochondrial superoxide production blocks three pathways of hyperglycaemic damage. Nature 404, 787-790 (2000).

32. N. Liu, J. Wu, L. Zhang, Z. Gao, Y. Sun, M. Yu, Y. Zhao, S. Dong, F. Lu, W. Zhang, Hydrogen Sulphide modulating mitochondrial morphology to promote mitophagy in endothelial cells under high-glucose and high-palmitate. J Cell Mol Med 21, 3190-3203 (2017).

33. R. A. Gioscia-Ryan, M. L. Battson, L. M. Cuevas, M. C. Zigler, A. L. Sindler, D. R. Seals, Voluntary aerobic exercise increases arterial resilience and mitochondrial health with aging in mice. Aging (Albany NY) 8, 2897-2914 (2016).

34. H. Y. Choi, J. H. Park, W. B. Jang, S. T. Ji, S. Y. Jung, Y. Kim da, S. Kang, Y. J. Kim, J. Yun, J. H. Kim, S. H. Baek, S. M. Kwon, High Glucose Causes Human Cardiac Progenitor Cell Dysfunction by Promoting Mitochondrial Fission: Role of a GLUT1 Blocker. Biomol Ther (Seoul) 24, 363-370 (2016). 
35. Y. Zhang, J. Feng, Q. Wang, S. Zhao, S. Yang, L. Tian, P. Meng, J. Li, H. Li, Hyperglycaemia Stress-Induced Renal Injury is Caused by Extensive Mitochondrial Fragmentation, Attenuated MKP1 Signalling, and Activated JNKCaMKII-Fis1 Biological Axis. Cell Physiol Biochem 51, 1778-1798 (2018).

36. O. C. Loson, Z. Song, H. Chen, D. C. Chan, Fis1, Mff, MiD49, and MiD51 mediate Drp1 recruitment in mitochondrial fission. Mol Biol Cell 24, 659-667 (2013).

37. B. Zinman, C. Wanner, J. M. Lachin, D. Fitchett, E. Bluhmki, S. Hantel, M. Mattheus, T. Devins, O. E. Johansen, H. J. Woerle, U. C. Broedl, S. E. Inzucchi, E.-R. O. Investigators, Empagliflozin, Cardiovascular Outcomes, and Mortality in Type 2 Diabetes. N Engl J Med 373, 2117-2128 (2015).

38. C. Wanner, S. E. Inzucchi, J. M. Lachin, D. Fitchett, M. von Eynatten, M. Mattheus, O. E. Johansen, H. J. Woerle, U. C. Broedl, B. Zinman, E.-R. O. Investigators, Empagliflozin and Progression of Kidney Disease in Type 2 Diabetes. N Engl J Med 375, 323-334 (2016).

39. M. Mizuno, A. Kuno, T. Yano, T. Miki, H. Oshima, T. Sato, K. Nakata, Y. Kimura, M. Tanno, T. Miura, Empagliflozin normalizes the size and number of mitochondria and prevents reduction in mitochondrial size after myocardial infarction in diabetic hearts. Physiol Rep 6, e13741 (2018).

40. Q. Wang, M. Zhang, G. Torres, S. Wu, C. Ouyang, Z. Xie, M. H. Zou, Metformin Suppresses Diabetes-Accelerated Atherosclerosis via the Inhibition of Drp1Mediated Mitochondrial Fission. Diabetes 66, 193-205 (2017).

41. H. Liu, H. Xiang, S. Zhao, H. Sang, F. Lv, R. Chen, Z. Shu, A. F. Chen, S. Chen, H. Lu, Vildagliptin improves high glucose-induced endothelial mitochondrial dysfunction via inhibiting mitochondrial fission. J Cell Mol Med 23, 798-810 (2019).

42. R. Yu, S. B. Jin, U. Lendahl, M. Nister, J. Zhao, Human Fis1 regulates mitochondrial dynamics through inhibition of the fusion machinery. EMBO J 38, (2019).

43. D. Bach, S. Pich, F. X. Soriano, N. Vega, B. Baumgartner, J. Oriola, J. R. Daugaard, J. Lloberas, M. Camps, J. R. Zierath, R. Rabasa-Lhoret, H. WallbergHenriksson, M. Laville, M. Palacin, H. Vidal, F. Rivera, M. Brand, A. Zorzano, Mitofusin-2 determines mitochondrial network architecture and mitochondrial metabolism. A novel regulatory mechanism altered in obesity. J Biol Chem 278, 17190-17197 (2003).

44. J. J. Lugus, G. A. Ngoh, M. M. Bachschmid, K. Walsh, Mitofusins are required for angiogenic function and modulate different signaling pathways in cultured endothelial cells. J Mol. Cell Cardiol 51, 885-893 (2011).

Acknowledgement: We gratefully acknowledge Ms. Cathy Paddock and Dr. Peter Newman for training and access to the ECIS instrument for TEER measurements. Funding: Dr. Puppala received support from GM089586. Dr. Hofeld is supported by R38HL143561. Dr. Hill receives support from HL128240 and GM067180. Dr. Widlansky receives support from HL128240, HL125409, HL144098, R38HL143561, and 
R61AT010680. Ms. Meacham receives support from the National Center for Advancing Translational Sciences, National Institutes of Health, Award Number TL1TR001437. Mr. Trykall was supported by T35HL0724485 and a participant the Medical College of Wisconsin's Molecular and Cellular Research Pathway. Author contributions: M.E.W. and R.B.H. oversaw the conception, design, and conduct of the study. M.K. was responsible for HMEC-1 data collection, processing and analysis and wrote portions of the manuscript. J.M.E. was responsible for pep213 data collection, processing and analysis and wrote portions of the manuscript. J.L. was responsible for human vessel data collection, processing, and analysis. M.C.H. supervised HMEC-1 data collection. K.A.M. was responsible for the pep213-scrambled data collection, processing, and analysis, wrote portions of the manuscript and made all final figures. M.E.W., V.K.P, and B.J.H. were responsible for clinical assessments and for collection of human arteriole samples. L.A.A. contributed to pep213-scrambled design. D.Z.T. conducted HMEC-1 experiments. All authors provided important intellectual input and approved the final version of the manuscript. The content is solely the responsibility of the authors and does not necessarily represent the official views of the NIH. Competing Interests: Drs. Hill and Widlansky receive significant support from research grant HL128240 directly relevant to this manuscript. The authors declare they have no affiliations with or involvement in any organization or entity with any financial interest, or non-financial interest in the subject matter or materials discussed in this manuscript. A provisional patent application has been filed for pep213. Data and materials availability: All data associated with this study are present in the paper, the Supplementary Materials, or available upon request. pep213 and pep213scr are available after completion of a materials transfer agreement. 


\section{Supplemental Methods: All methods are identical to those described in the main text .}

5' to 3' sequences for siRNA to Fis 1 and Drp1 were the following:

Fis1:

A: rGrGrUrGrCrGrGrArGrCrArArGrUrArCrArArUrGrArUrGAC

B: rArCrUrArCrCrGrGrCrUrCrArArGrGrArArUrArCrGrArGAA

C: rArCrArGrUrArGrArCrUrGrUrArGrUrGrUrGrArGrGrCrUCG

Drp1:

A: rArGrArGrUrGrUrArArCrUrGrArUrUrCrArArUrCrCrGrUGA

B: rArGrGrArUrArUrUrGrArGrCrUrUrCrArArArUrCrArGrAGA

C: rCrCrCrUrUrArArArCrUrGrArGrUrCrArArGrArUrCrUrGAA

\section{Supplemental Results:}

Consistent with excessive mitochondrial fission in impairing NO-dependent vasodilation in the diabetic endothelium, we previously reported similar findings upon genetic silencing of the mitochondrial fission mechanoenzyme Drp1 (8). In those studies, siRNA to Drp1 significantly reduces Drp1 expression in human arterioles and prevented LG-induced impairment endothelium-dependent vasodilation in resistance arteries from healthy humans. ${ }^{8}$ In the current study, we found that genetic silencing of Drp1 also prevented high glucose-induced impairment of endothelium-dependent vasodilation, (Supplemental Figure 2, $\mathrm{N}=6, \mathrm{P}<0.0001$ overall vs. all other exposures), which was completely abolished by L-NAME but not papaverine (data not shown). Additionally, DAF-2 DA fluorescence was also significantly increased in vessels from healthy individuals exposed to high glucose (Supplemental Figure 3A; $n=9, P=0.02$ overall $\mathrm{P}<0.05$ for Drp1 siRNA vs. scrambled control, scrambled control + LNAME, and Drp1 siRNA + L-NAME) and low glucose (Supplemental Figure 3B, $\mathrm{n}=5, \mathrm{P}=0.003$ overall, $\mathrm{P}<0.04$ Fis 1 siRNA vs. all other exposures). L-NAME completely abolished the increases in DAF2-DA fluorescence in both cases. Additionally, transfection with Drp1 siRNA showed a trend toward improved endothelium-dependent vasodilation in vessels from humans with type 2 diabetes (Supplemental Figure 4). 


\begin{tabular}{|c|c|c|c|c|}
\hline \multicolumn{5}{|c|}{$\begin{array}{l}\text { Supplemental Table 1: Demographic, clinical, and medication information and in vivo vascular function for } \\
16 \text { subjects with resistance arterioles that underwent Fis1 siRNA transfection and studied for vasodilation } \\
\text { in response to acetylcholine }\end{array}$} \\
\hline & $\begin{array}{c}\text { Non-Diabetic } \\
(L G ; n=6)\end{array}$ & $\begin{array}{c}\text { Non-Diabetic } \\
(H G ; n=5)\end{array}$ & $\begin{array}{c}\text { Type } 2 \text { DM } \\
(n=6)\end{array}$ & p-value \\
\hline Age & $35 \pm 17$ & $58 \pm 5$ & $61 \pm 10$ & 0.01 \\
\hline Sex (\#female) & 3 & 3 & 0 & 0.12 \\
\hline Smoking Status (\#never) & 1 & 1 & 3 & 0.03 \\
\hline History of Hypertension & 0 & 0 & 5 & 0.0002 \\
\hline History of High Cholesterol & 0 & 0 & 5 & \\
\hline Body mass Index (kg/m2) & $28 \pm 5$ & $26 \pm 3$ & $29 \pm 5$ & 0.80 \\
\hline Waist Circumference (cm) & $95 \pm 18$ & $88 \pm 10$ & $109 \pm 4$ & 0.18 \\
\hline Systolic Blood Pressure (mmHg) & $120 \pm 17$ & $126 \pm 11$ & $126 \pm 11$ & 0.69 \\
\hline Diastolic Blood Pressure (mmHg) & $72 \pm 10$ & $74 \pm 11$ & $74 \pm 8$ & 0.85 \\
\hline Fasting Glucose (mg/dL) & $83 \pm 11$ & $82 \pm 7$ & $174 \pm 35$ & $<0.0001$ \\
\hline Hemoglobin A1C (\%) & $5.3 \pm 0.3$ & $5.4 \pm 0.2$ & $8.3 \pm 1.8$ & 0.0009 \\
\hline Creatinine (mg/dL) & $0.82 \pm 0.16$ & $0.92 \pm 0.18$ & $0.95 \pm 0.19$ & 0.25 \\
\hline Total Cholesterol (mg/dL) & $204 \pm 33$ & $195 \pm 16$ & $174 \pm 41$ & 0.35 \\
\hline HDL Cholesterol & $71 \pm 15$ & $86 \pm 19$ & $49 \pm 6$ & 0.009 \\
\hline LDL Cholesterol (mg/dL) & $115 \pm 34$ & $95 \pm 18$ & $84 \pm 31$ & 0.21 \\
\hline \multicolumn{5}{|l|}{ Medications ( $\%$ on Therapy) } \\
\hline Biguanide & 0 & 0 & 83 & \\
\hline Sulfonylurea & 0 & 0 & 83 & \\
\hline Thiazoladinedione & 0 & 0 & 0 & \\
\hline DPP4 inhibitor & 0 & 0 & 33 & \\
\hline GLP-1 Agonist & 0 & 0 & 0 & \\
\hline Insulin & 0 & 0 & 0 & \\
\hline HMG CoA reductase Inhibitor & 0 & 0 & 100 & \\
\hline ACE Inhibitor & 0 & 0 & 83 & \\
\hline Angiotensin II Receptor Blocker & 0 & 0 & 17 & \\
\hline GLP1 Agonist & 0 & 0 & 17 & \\
\hline SGLT2 Inhibitor & 0 & 0 & 0 & \\
\hline
\end{tabular}




\begin{tabular}{|c|c|c|c|}
\hline \multicolumn{4}{|c|}{$\begin{array}{l}\text { Supplemental Table 2: Demographic, clinical, and medication information and in vivo } \\
\text { vascular function for } 17 \text { subjects with resistance arterioles that underwent Fis1 siRNA } \\
\text { transfection and studied for biological NO production using DAF2DA fluorescence. }\end{array}$} \\
\hline & $\begin{array}{c}\text { Non-DM } \\
(L G ; n=8)\end{array}$ & $\begin{array}{c}\text { Non-DM } \\
(\mathrm{HG} ; \mathrm{n}=9)\end{array}$ & p-value \\
\hline Age & $42 \pm 16$ & $35 \pm 14$ & 0.54 \\
\hline Sex (\#female) & 5 & 8 & 0.35 \\
\hline Smoking Status (\#never) & 3 & 2 & 0.38 \\
\hline History of Hypertension & 0 & 0 & - \\
\hline History of High Cholesterol & 0 & 0 & - \\
\hline Body mass Index (kg/m2) & $23 \pm 3$ & $23 \pm 6$ & 0.89 \\
\hline Waist Circumference (cm) & $85 \pm 11$ & $82 \pm 17$ & 0.78 \\
\hline Systolic Blood Pressure (mmHg) & $125 \pm 9$ & $111 \pm 12$ & 0.02 \\
\hline Diastolic Blood Pressure (mmHg) & $68 \pm 5$ & $68 \pm 12$ & 0.82 \\
\hline Fasting Glucose (mg/dL) & $85 \pm 3$ & $89 \pm 9$ & 0.50 \\
\hline Hemoglobin A1C (\%) & $5 \pm 0.2$ & $5.2 \pm 0.3$ & 0.27 \\
\hline Creatinine $(\mathrm{mg} / \mathrm{dL})$ & $0.8 \pm 0.1$ & $0.74 \pm 0.11$ & 0.12 \\
\hline Total Cholesterol (mg/dL) & $197 \pm 31$ & $171 \pm 22$ & 0.14 \\
\hline HDL Cholesterol & $70 \pm 16$ & $63 \pm 23$ & 0.81 \\
\hline LDL Cholesterol (mg/dL) & $109 \pm 23$ & $89 \pm 22$ & 0.12 \\
\hline
\end{tabular}

\section{DM-Diabetes}




\begin{tabular}{|c|c|c|c|}
\hline \multicolumn{4}{|c|}{$\begin{array}{l}\text { Supplemental Table 3: Demographic, clinical, and medication information and in } \\
\text { vivo vascular function for } 13 \text { subjects with resistance arterioles that underwent } \\
\text { Drp1 siRNA transfection and studied for vasodilation in response to acetylcholine. }\end{array}$} \\
\hline & $\begin{array}{c}\begin{array}{c}\text { Non-Diabetic } \\
(\mathrm{HG} ; \mathrm{n}=5)\end{array} \\
\end{array}$ & $\begin{array}{c}\text { Type 2 DM } \\
(n=4)\end{array}$ & p-value \\
\hline Age & $33 \pm 11$ & $46 \pm 15$ & 0.43 \\
\hline Sex (\#female) & 2 & 3 & 0.29 \\
\hline Smoking Status (\#never) & 5 & 2 & 0.49 \\
\hline History of Hypertension & 0 & 3 & 0.01 \\
\hline History of High Cholesterol & 0 & 2 & 0.20 \\
\hline Body mass Index (kg/m2) & $27 \pm 7$ & $30 \pm 5$ & 0.49 \\
\hline Waist Circumference (cm) & $89 \pm 19$ & $95 \pm 12$ & 0.75 \\
\hline Systolic Blood Pressure (mmHg) & $119 \pm 20$ & $131 \pm 33$ & 0.96 \\
\hline Diastolic Blood Pressure (mmHg) & $74 \pm 15$ & $79 \pm 22$ & 0.95 \\
\hline Fasting Glucose (mg/dL) & $71 \pm 9$ & $142 \pm 55$ & 0.002 \\
\hline Hemoglobin A1C (\%) & $5.2 \pm 0.5$ & $8.4 \pm 1.0$ & $<0.0001$ \\
\hline Creatinine $(\mathrm{mg} / \mathrm{dL})$ & $0.89 \pm 0.23$ & $0.82 \pm 0.22$ & 0.40 \\
\hline Total Cholesterol (mg/dL) & $179 \pm 18$ & $163 \pm 16$ & 0.09 \\
\hline HDL Cholesterol & $74 \pm 27$ & $56 \pm 3$ & 0.39 \\
\hline LDL Cholesterol (mg/dL) & $87 \pm 13$ & $90 \pm 19$ & 0.40 \\
\hline \multicolumn{4}{|l|}{ Medications (\% on Therapy) } \\
\hline Biguanide & 0 & 75 & \\
\hline Sulfonylurea & 0 & 25 & \\
\hline Thiazoladinedione & 0 & 0 & \\
\hline DPP4 inhibitor & 0 & 0 & \\
\hline GLP-1 Agonist & 0 & 0 & \\
\hline Insulin & 0 & 25 & \\
\hline HMG CoA reductase Inhibitor & 0 & 50 & \\
\hline ACE Inhibitor & 0 & 25 & \\
\hline Angiotensin II Receptor Blocker & 0 & 0 & \\
\hline SGLT2 Inhibitor & 0 & 0 & \\
\hline
\end{tabular}

DM-Diabetes 


\begin{tabular}{|c|c|c|c|}
\hline \multicolumn{4}{|c|}{$\begin{array}{l}\text { Supplemental Table 4: Demographic, clinical, and medication information and in } \\
\text { vivo vascular function for } 16 \text { subjects with resistance arterioles that underwent Drp1 } \\
\text { siRNA transfection and studied for biological NO production using DAF2DA } \\
\text { fluorescence. }\end{array}$} \\
\hline & $\begin{array}{c}\text { Non-Diabetic } \\
(\text { LG; } n=5)\end{array}$ & $\begin{array}{l}\text { Type } 2 \text { DM } \\
\text { (HG; } n=9)\end{array}$ & p-value \\
\hline Age & $29 \pm 9$ & $42 \pm 14$ & 0.27 \\
\hline Sex (\#female) & 3 & 6 & 0.37 \\
\hline Smoking Status (\#never) & 3 & 2 & 0.36 \\
\hline History of Hypertension & 0 & 0 & na \\
\hline History of High Cholesterol & 0 & 0 & na \\
\hline Body mass Index (kg/m2) & $25 \pm 6$ & $29 \pm 6$ & 0.26 \\
\hline $\begin{array}{l}\text { Waist Circumference }(\mathrm{cm}) \\
\text { Systolic Blood Pressure }\end{array}$ & $86 \pm 16$ & $94 \pm 15$ & 0.70 \\
\hline $\begin{array}{l}(\mathrm{mmHg}) \\
\text { Diastolic Blood Pressure }\end{array}$ & $116 \pm 10$ & $126 \pm 12$ & 0.61 \\
\hline$(\mathrm{mmHg})$ & $71 \pm 11$ & $71 \pm 10$ & 0.91 \\
\hline Fasting Glucose (mg/dL) & $82 \pm 13$ & $87 \pm 9$ & 0.45 \\
\hline Hemoglobin A1C (\%) & $5.1 \pm 0.1$ & $5.2 \pm 0.3$ & 0.42 \\
\hline Creatinine (mg/dL) & $0.81 \pm 0.15$ & $0.86 \pm 0.21$ & 0.53 \\
\hline Total Cholesterol (mg/dL) & $170 \pm 35$ & $178 \pm 27$ & 0.72 \\
\hline HDL Cholesterol & $67 \pm 10$ & $61 \pm 12$ & 0.74 \\
\hline LDL Cholesterol (mg/dL) & $86 \pm 29$ & $97 \pm 20$ & 0.76 \\
\hline
\end{tabular}

DM-Diabetes 
Supplemental Table 5: Demographic, clinical, and medication information and in vivo vascular function for 10 subjects with resistance arterioles exposed to pep213 and studied for vasodilation in response to acetylcholine in Figure 7

\begin{tabular}{|c|c|c|c|}
\hline & $\begin{array}{c}\text { Non-DM } \\
(\mathrm{HG} ; \mathrm{n}=6)\end{array}$ & T2DM $(n=4)$ & p-value \\
\hline Age & $35 \pm 14$ & $46 \pm 15$ & 0.02 \\
\hline Sex (\#female) & 1 & 2 & 0.29 \\
\hline Smoking Status (\#never) & 5 & 1 & 0.49 \\
\hline History of Hypertension & 0 & 3 & 0.01 \\
\hline History of High Cholesterol & 0 & 3 & 0.20 \\
\hline Body mass Index (kg/m2) & $27 \pm 7$ & $30 \pm 5$ & 0.007 \\
\hline Waist Circumference (cm) & $89 \pm 19$ & $95 \pm 12$ & 0.04 \\
\hline $\begin{array}{l}\text { Systolic Blood Pressure (mmHg) } \\
\text { Diastolic Blood Pressure } \\
\text { (mmHa) }\end{array}$ & $\begin{array}{l}117 \pm 7 \\
70+5\end{array}$ & $\begin{array}{l}125 \pm 16 \\
77+7\end{array}$ & 0.32 \\
\hline Fasting Glucose (mg/dL) & $92 \pm 6$ & $129 \pm 36$ & 0.04 \\
\hline Hemoglobin A1C (\%) & $5.0 \pm 0.3$ & $8.0 \pm 2.4$ & 0.01 \\
\hline Creatinine (mg/dL) & $0.92 \pm 0.16$ & $0.77 \pm 0.07$ & 0.11 \\
\hline Total Cholesterol (mg/dL) & $179 \pm 35$ & $179 \pm 55$ & 0.98 \\
\hline HDL Cholesterol & $55 \pm 19$ & $50 \pm 18$ & 0.67 \\
\hline LDL Cholesterol (mg/dL) & $110 \pm 22$ & $104 \pm 41$ & 0.79 \\
\hline \multicolumn{4}{|l|}{ Medications (\% on Therapy) } \\
\hline Biguanide & 0 & 100 & \\
\hline Sulfonylurea & 0 & 50 & \\
\hline Thiazoladinedione & 0 & 0 & \\
\hline GLP-1 Agonist & 0 & 25 & \\
\hline DPP4 inhibitor & 0 & 0 & \\
\hline Insulin & 0 & 25 & \\
\hline HMG CoA reductase Inhibitor & 0 & 50 & \\
\hline ACE Inhibitor & 0 & 25 & \\
\hline Angiotensin II Receptor Blocker & 0 & 25 & \\
\hline SGLT2 Inhibitor & 0 & 0 & \\
\hline
\end{tabular}

DM-Diabetes 
Supplemental Table 6: Demographic, clinical, and medication information and in vivo vascular function for 9 subjects with resistance arterioles exposed to TATpep213and scrambled peptide-TAT and studied for vasodilation in response to acetylcholine in Figure 8

\begin{tabular}{|lccr|}
\hline & $\begin{array}{c}\text { Non-DM } \\
(\mathbf{H G} \text {; } \mathbf{n = 5})\end{array}$ & T2DM (n=4) & p-value \\
\hline Age & $54 \pm 19$ & $46 \pm 15$ & 0.41 \\
Sex (\#female) & 4 & 2 & 0.2887 \\
Smoking Status (\#smoker) & 0 & 0 & - \\
History of Hypertension & 1 & 1 & 0.72 \\
History of High Cholesterol & 0 & 1 & 0.56 \\
Body mass Index (kg/m2) & $29 \pm 6$ & $35 \pm 10$ & 0.27 \\
Medications (\% on Therapy) & & & \\
\multicolumn{1}{r}{ Angiotensin II Receptor Blocker } & 0 & 0 & - \\
\hline
\end{tabular}

DM-Diabetes

\section{Supplemental Figures:}

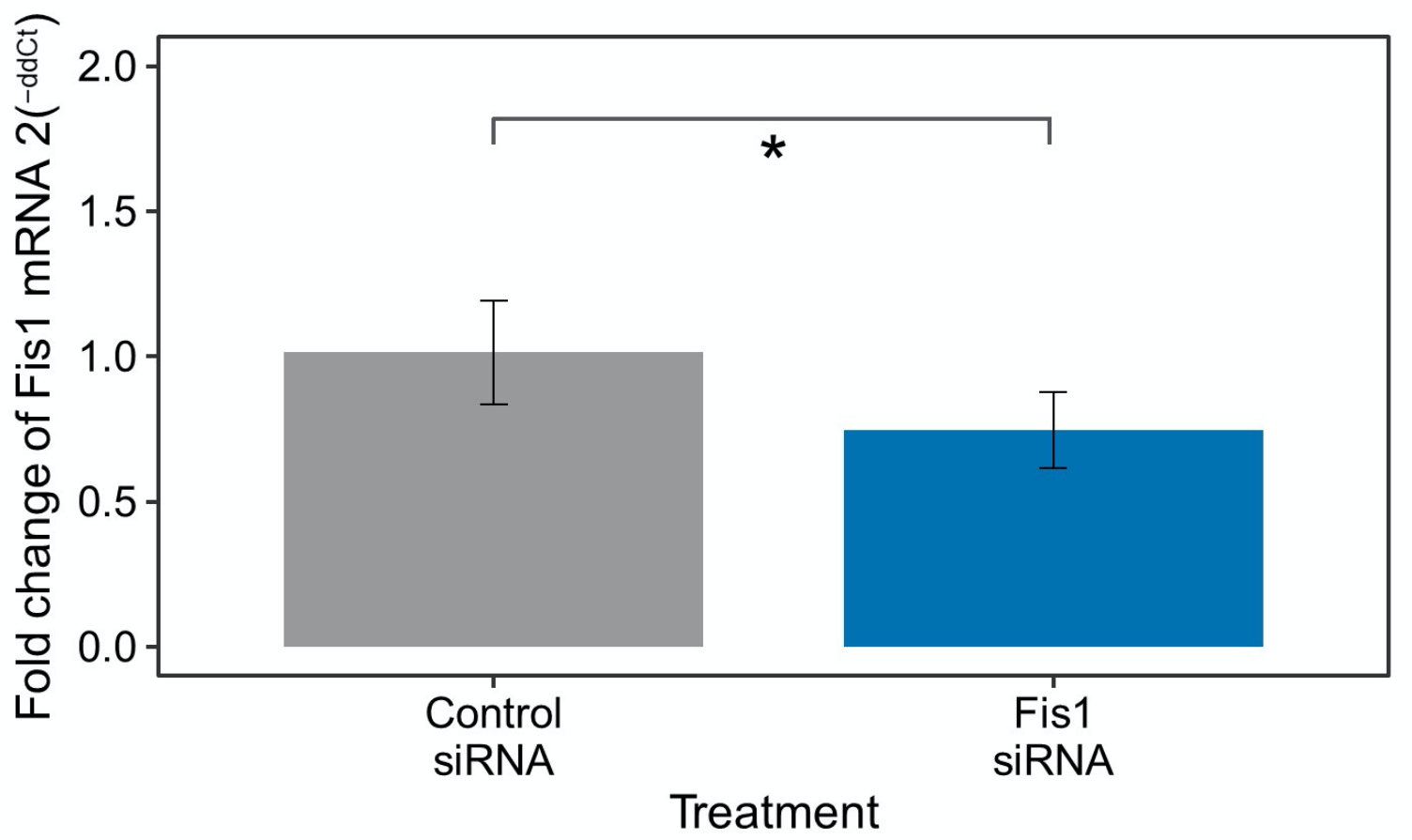

Supplemental Figure 1: Fis1 knockdown efficiency in human arterioles with siRNA treatment. Fis 1 levels were significantly reduced $(n=4, p<0.05)$ in human arterioles transfected with siRNA Fis 1 compared to arterioles transfected with scrambled control siRNA. 


\section{High Glucose}

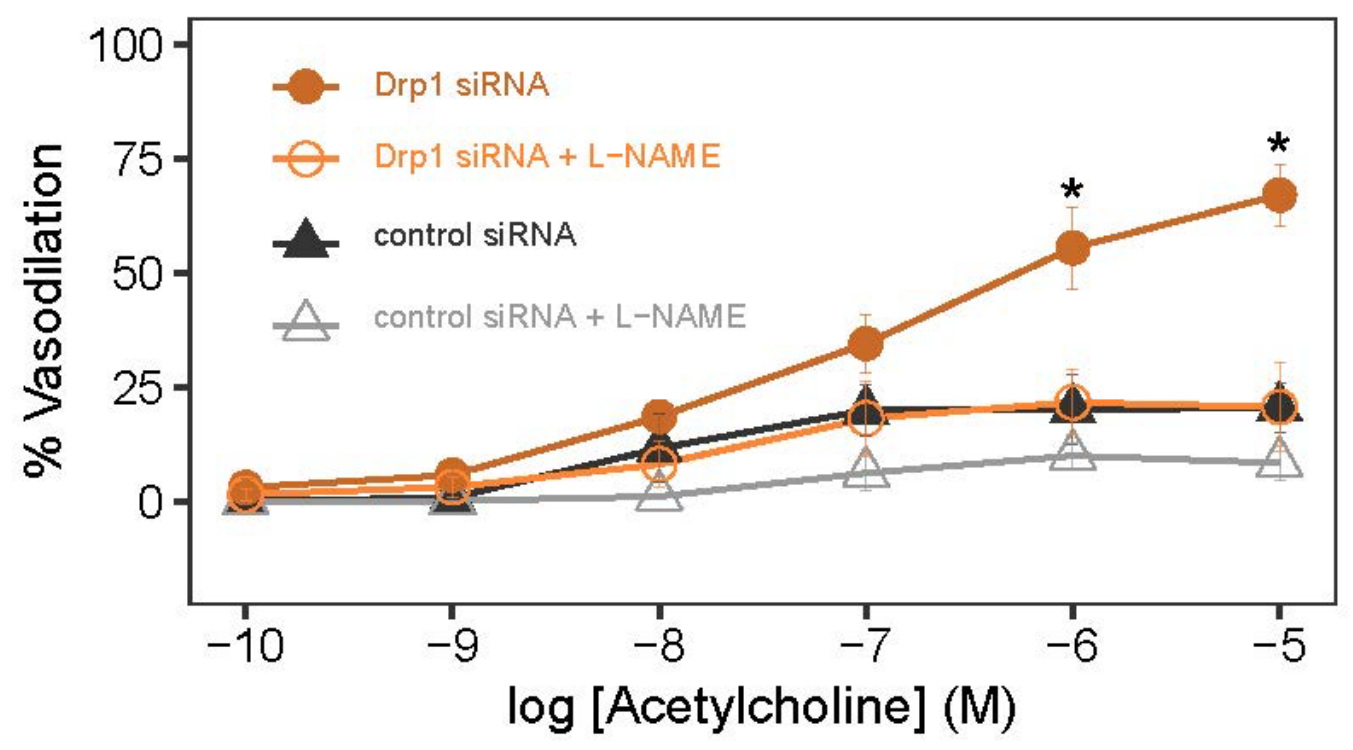

Supplemental Figure 2: Transfection with Drp1 siRNA protected against high glucoseinduced impairment in endothelium-dependent vasodilation. $(\mathrm{P}<0.0001$ overall, $* \mathrm{P} \leq 0.0001$ at indicated Ach doses for control siRNA vs Drp1 siRNA, n=6). L-NAME abolished this improvement of vasodilation under Drp1 knockdown conditions $(\mathrm{P}<0.0001$ for Drp1 siRNA vs.Drp1 siRNA +L-NAME).
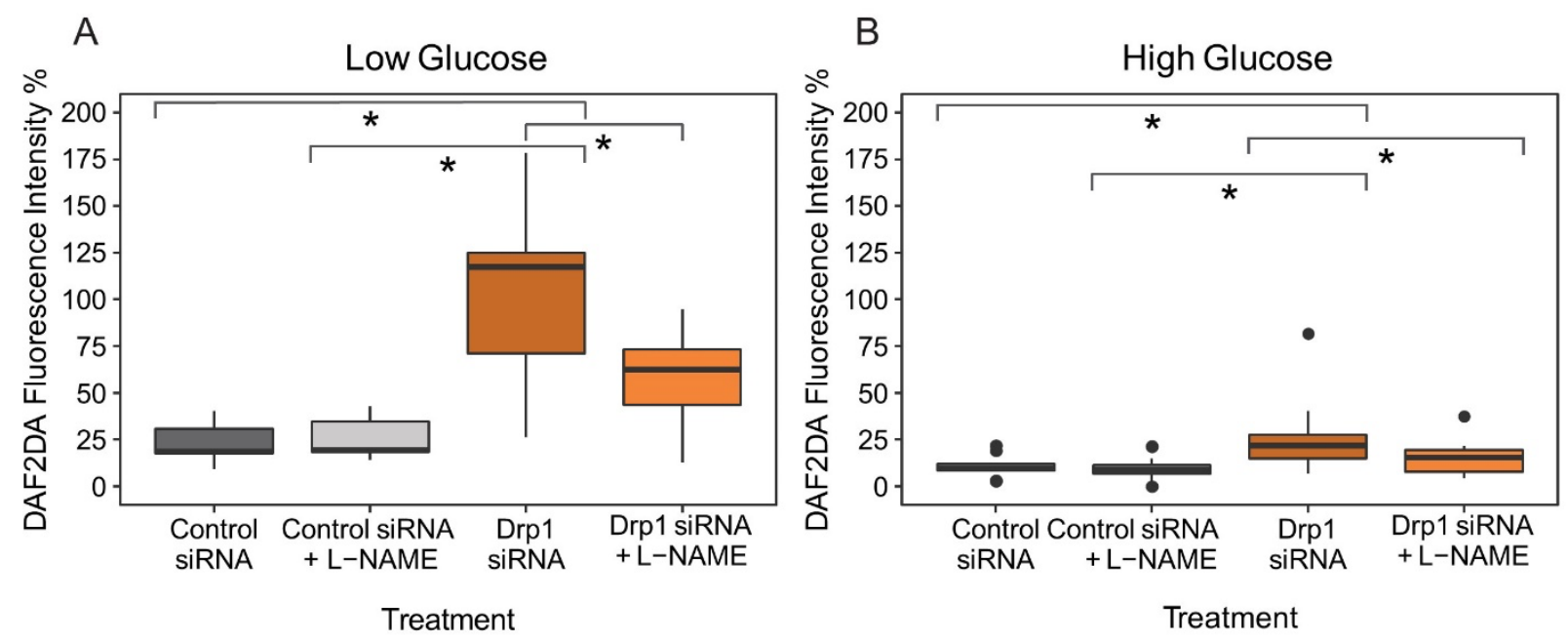

Supplemental Figure 3: Knockdown of Drp1 expression with Drp1 siRNA protected against reductions in nitric oxide (NO) bioavailability in arterioles from healthy human arterioles exposed to high or low glucose conditions. (A) High glucose (HG, 33mM, six-hour exposure): $\mathrm{n}=9, \mathrm{P}=0.02$ overall; $\mathrm{P}<0.05$ for Drp1 siRNA vs. all other exposures. (B) Low glucose (LG, 2.5mM, two-hour exposure): $\mathrm{n}=5$; overall $\mathrm{P}=0.003, \mathrm{P}=0.03$ or less for Drp1 siRNA vs. all other exposures. 


\section{Diabetes Mellitus}

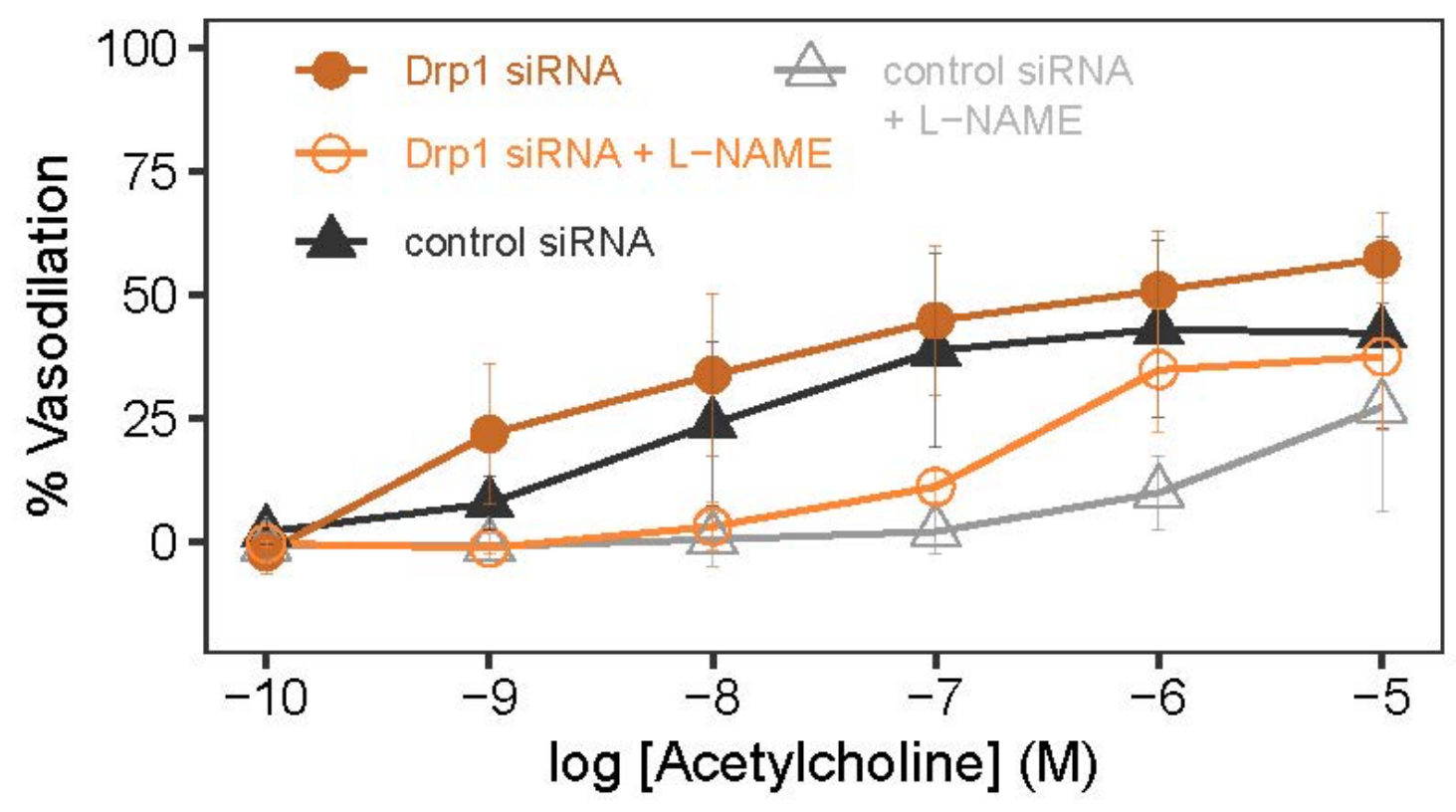

Supplemental Figure 4: Suppression of Drp1 expression in arterioles from humans with T2DM using Drp1 siRNA tended to reverse impaired endothelium-dependent vasodilation $(\mathrm{n}=4, \mathrm{P}=0.076)$. 


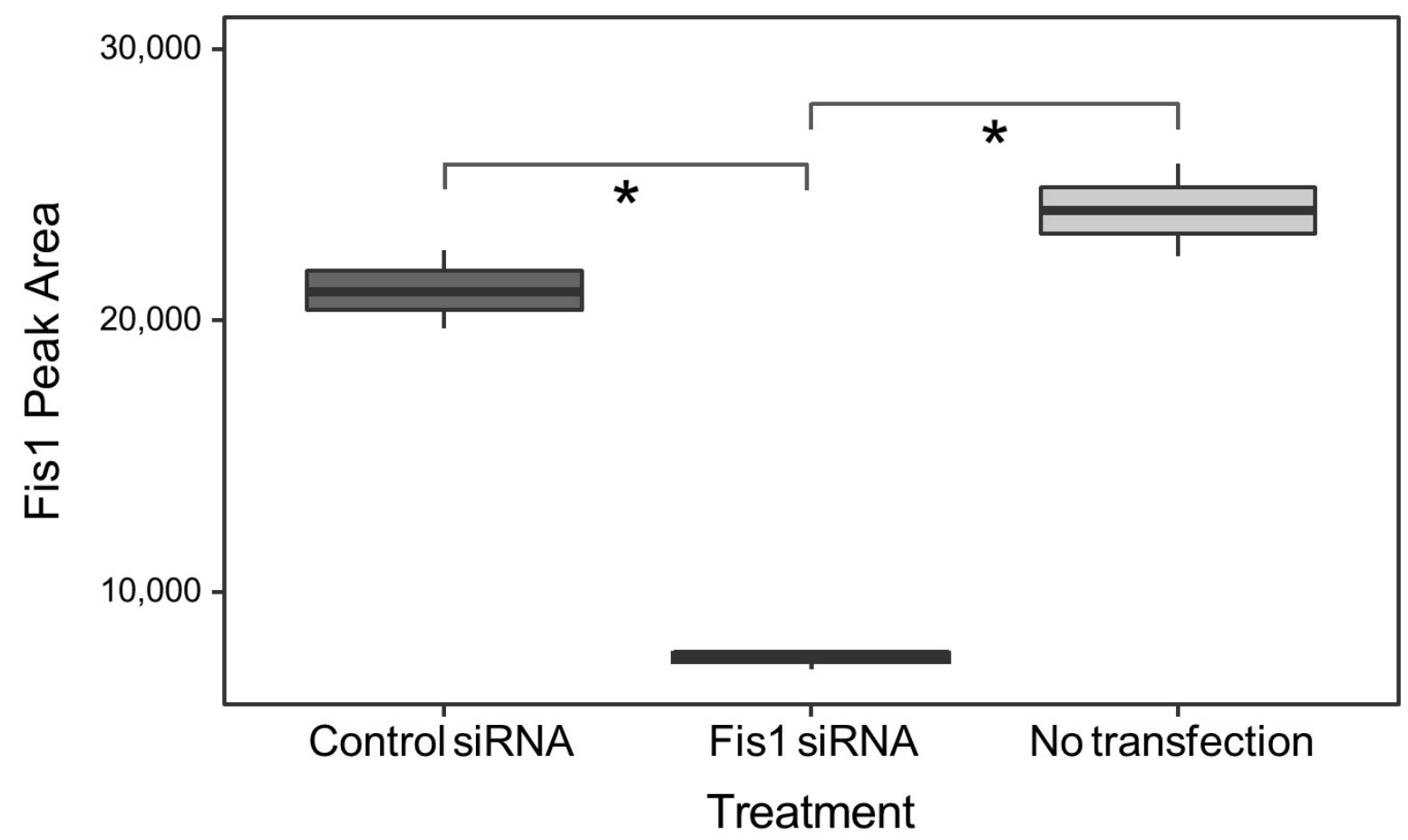

Supplemental Figure 5: Fis1 knockdown efficiency in HMEC-1 cells with siRNA treatment. Fis 1 levels were significantly reduced $(n=3$, overall $\mathrm{P}<0.0001)$ in HMEC-1 cells transfected with Fis1 siRNA Fis1 compared to cells transfected with scrambled control siRNA $(\mathrm{P}=0.0002)$ and non-transfected HMEC-1 cells $(\mathrm{P}=0.0001)$. 
A A23187

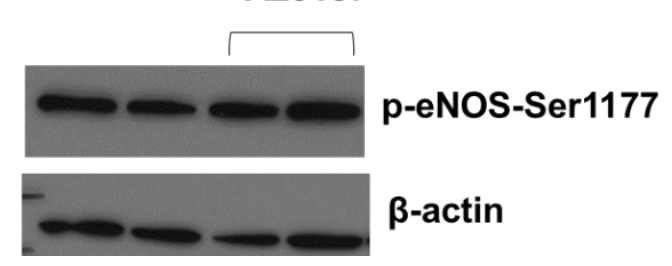

B
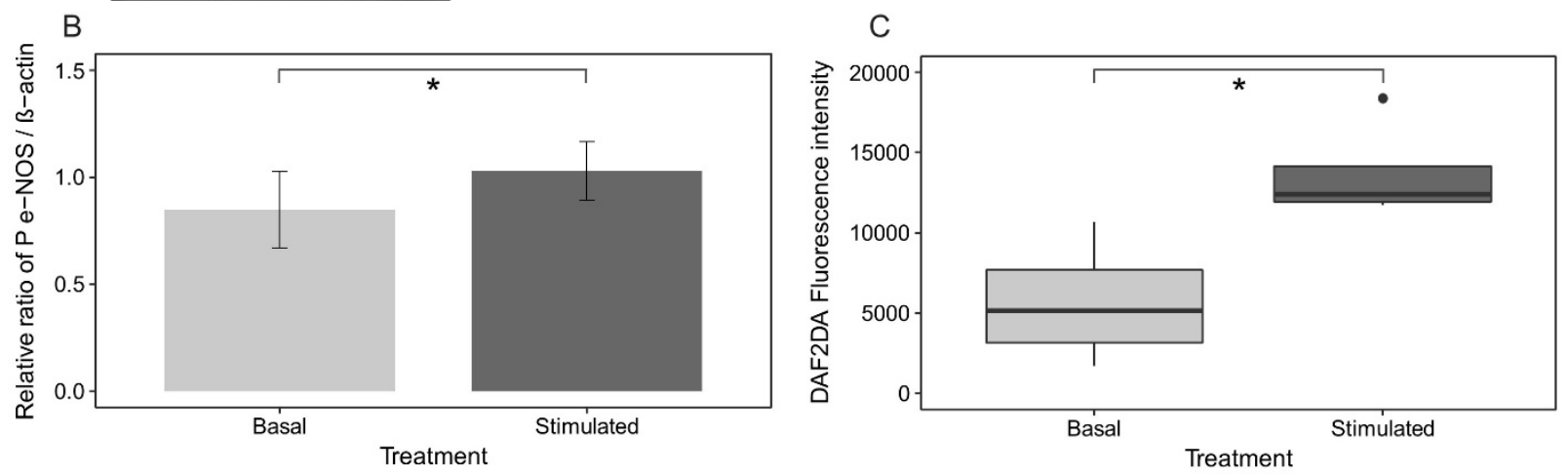

Supplemental Figure 6: Phosphorylation of eNOS at Ser1177 and NO production increased in HMEC-1 cells upon activation with $\mathrm{Ca}^{+2}$ ionophore $\mathbf{A 2 3 1 8 7}$ (A) representative western blot of p-eNOS-Ser1177) and $\beta$-actin with and without addition of $5 \mu \mathrm{M}$ A23187.(B)

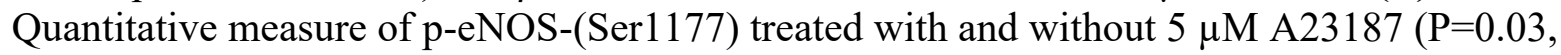
$\mathrm{n}=6$, data are mean $\pm \mathrm{SD})$. (C) NO production as measured using DAF2-DA $(5 \mu \mathrm{M})$ increased upon addition of $\mathrm{A} 23187(\mathrm{n}=4, \mathrm{P}=0.02)$.

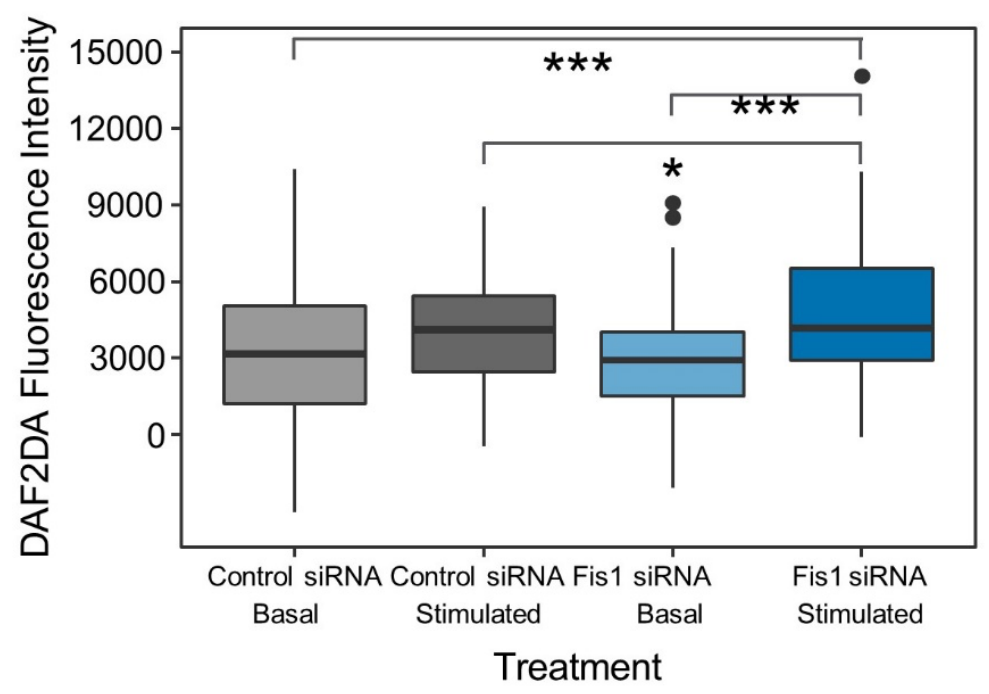

Supplemental Figure 7: Fis1 knockdown improved NO production in immortalized cultured human microvascular endothelial cells (HMEC-1) transfected with Fis1 siRNA. HMEC-1 cells were treated with vehicle control (basal) or $5 \mu \mathrm{M}$ A23187 for $2 \mathrm{hrs}$ followed by a 15-minute incubation with DAF2-DA $(5 \mathrm{uM})$ prior to measurement of fluorescence intensity, $\mathrm{n}=7, \mathrm{P}<0.0001$ overall; siFis 1 basal vs siFis1 stimulated- $\mathrm{P}<0.0001$; siRNA basal vs siFis 1 stimulated- $\mathrm{P}=0.0003$; siFis1 stimulated vs siRNA stimulated- $\mathrm{P}=0.02$ ). 


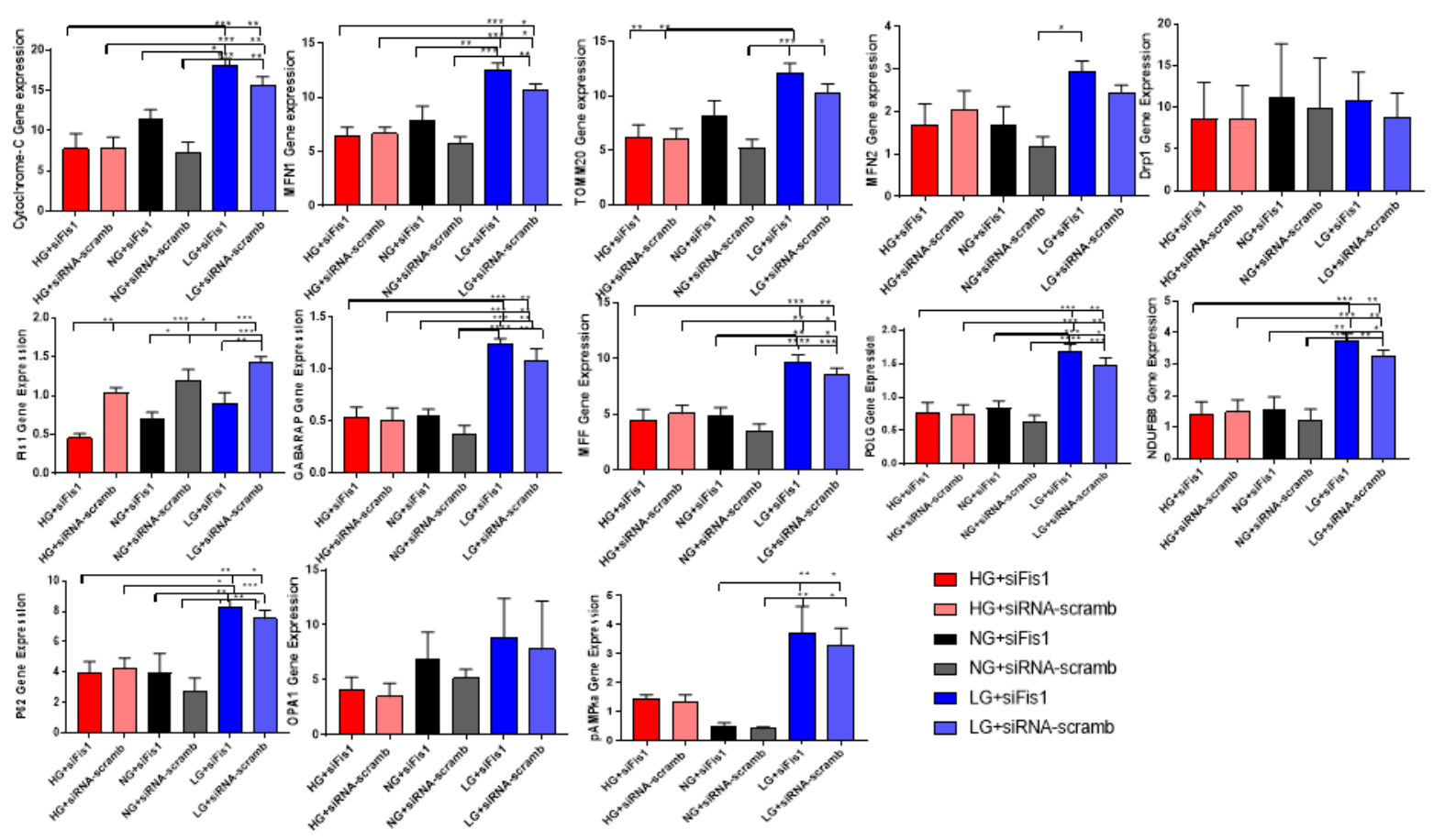

Supplemental Figure 8: Molecular inhibition of Fis1 under high glucose (33mM) and low glucose $(2.5 \mathrm{mM})$ conditions did not alter the expression of other mitochondrial proteins. The expression of selected mitochondrial proteins was measured from immortalized HMEC-1 cells transfected with siRNA Fis1 or scrambled siRNA and pre-incubated with different glucose conditions: high glucose (HG, 33mM for six hours), normal glucose (NG, 5mM for 2 hours) and low glucose ( $\mathrm{LG}, 2.5 \mathrm{mM}$ for 2 hours). The expression of each protein was normalized to total protein across the samples. ( $\mathrm{n}=4-10$ for individual proteins). While there are differences in expression of some mitochondrial proteins seen when comparing different glucose concentration exposures, knockdown of Fis1 expression with siRNA did not affect expression of any mitochondrial protein except for Fis 1 . Statistically significant differences are indicated by $*$ for $\mathrm{p}<0.05, * *$ for $\mathrm{p}<0.01, * * *$ for $\mathrm{p}<0.001$, and $* * * *$ for $\mathrm{p}<0.0001$. 